\title{
Quantum and affine Schubert calculus and Macdonald polynomials
}

\author{
Avinash J. Dalal 11 and Jennifer Morse $2^{2}$ \\ Drexel University \\ Department of Mathematics \\ Philadelphia, PA 19104
}

\begin{abstract}
We definitively establish that the theory of symmetric Macdonald polynomials aligns with quantum and affine Schubert calculus using a discovery that distinguished weak chains can be identified by chains in the strong (Bruhat) order poset on the type- $A$ affine Weyl group. We construct two one-parameter families of functions that respectively transition positively with Hall-Littlewood and Macdonald's $P$-functions, and specialize to the representatives for Schubert classes of homology and cohomology of the affine Grassmannian. Our approach leads us to conjecture that all elements in a defining set of 3-point genus 0 Gromov-Witten invariants for flag manifolds can be formulated as strong covers.
\end{abstract}

\section{Introduction}

The Macdonald polynomial basis for the ring $\Lambda$ of symmetric functions is found at the root of many exciting projects spanning topics such as double affine Hecke algebras, quantum relativistic systems, diagonal harmonics, and Hilbert schemes on points in the plane. The transition matrix between Macdonald and Schur functions has been intensely studied from a combinatorial, representation theoretic, and algebraic geometric perspective since the time Macdonald conjectured [Mac88] that the coefficients in the expansion

$$
H_{\mu}(x ; q, t)=\sum_{\lambda} K_{\lambda \mu}(q, t) s_{\lambda}
$$

are positive sums of monomials in $q$ and $t$ - that is, $K_{\lambda \mu}(q, t) \in \mathbb{N}[q, t]$.

The Kostka-Foulkes polynomials are the special case $K_{\lambda \mu}(0, t)$. These appear in contexts such as Hall-Littlewood polynomials [Gre55], affine Kazhdan-Lusztig theory [Lus81], and affine tensor product multiplicities [NY97]. Kostka-Foulkes polynomials also encode the dimensions of bigraded $S_{n}$-modules [GP92]. They were combinatorially characterized by Lascoux and Schützenberger [LS78] who associated a non-negative integer statistic called cocharge to each semi-standard Young tableau and proved that

$$
K_{\lambda \mu}(0, t)=\sum_{T \in S S Y T(\lambda, \mu)} t^{\operatorname{cocharge}(T)},
$$

\footnotetext{
${ }^{1}$ Supported by the NSF grants DMS-1001898, DMS-1301695.

${ }^{2}$ Supported by the NSF grants DMS-1001898, DMS-1301695, and a Simons Fellowship.
} 
summing over tableaux of shape $\lambda$ and weight $\mu$. Despite the prevelance of concrete results for the $K_{\lambda \mu}(0, t)$, it was a big effort even to establish polynomiality for general $K_{\lambda \mu}(q, t)$ and the geometry of Hilbert schemes was ultimately needed to prove positivity [Hai01]. A formula in the spirit of (2) still remains a complete mystery.

One study of Macdonald polynomials [LLM03] uncovered a manifestly $t$-Schur positive construction for polynomials $A_{\mu}^{(k)}(x ; t)$, conjectured to be a basis for the subspace $\Lambda_{(k)}^{t}$ in a filtration $\Lambda_{(1)}^{t} \subset \Lambda_{(2)}^{t} \subset \cdots \subset \Lambda_{(\infty)}^{t}$ of $\Lambda$ with the compelling feature that for every partition $\mu$ where $\mu_{1} \leq k$,

$$
H_{\mu}(x ; q, t)=\sum_{\lambda: \lambda_{1} \leq k} K_{\lambda \mu}^{k}(q, t) A_{\lambda}^{(k)}(x ; t) \quad \text { for some } \quad K_{\lambda \mu}^{k}(q, t) \in \mathbb{N}[q, t] .
$$

Assuming this conjecture, since $A_{\lambda}^{(k)}(x ; t)$ is a $t$-positive sum of Schur functions, the Macdonald/Schur transition matrices factor over $\mathbb{N}[q, t]$. The construction of $A_{\mu}^{(k)}(x ; t)$ is extremely intricate and the conjectures remain unproven as a consequence. Nevertheless, their study inspired discoveries in representation theory [Hai08] and suggested a connection between the theory of Macdonald polynomials and quantum and affine Schubert calculus.

The affine Grassmannian of $G=S L(n, \mathbb{C})$ is given by $\mathrm{Gr}=G(\mathbb{C}((t))) / G(\mathbb{C}[[t]])$, where $\mathbb{C}[[t]]$ is the ring of formal power series and $\mathbb{C}((t))=\mathbb{C}[[t]]\left[t^{-1}\right]$ is the ring of formal Laurent series. Quillen (unpublished) and Garland and Raghunathan [GR75] showed that Gr is homotopy-equivalent to the group $\Omega S U(n, \mathbb{C})$ of based loops into $S U(n, \mathbb{C})$. The homology $H_{*}(\mathrm{Gr})$ and cohomology $H^{*}(\mathrm{Gr})$ thus have dual Hopf algebra structures which, using results of [Bot58], can be explicitly identified by a subring $\Lambda^{(n)}$ and a quotient $\Lambda_{(n)}$ of $\Lambda$.

On one hand, the algebraic nil-Hecke ring construction of Kostant and Kumar [KK86] and the work of Peterson [Pet97] developed the study of Schubert bases associated to Schubert cells of Gr in the Bruhat decomposition of $G(\mathbb{C}((t)))$,

$$
\left\{\xi^{w} \in H^{*}(\mathrm{Gr}, \mathbb{Z}) \mid w \in \tilde{S}_{n}^{0}\right\} \quad \text { and } \quad\left\{\xi_{w} \in H_{*}(\mathrm{Gr}, \mathbb{Z}) \mid w \in \tilde{S}_{n}^{0}\right\},
$$

indexed by Grassmannian elements of the affine Weyl group $\tilde{A}_{n-1}$. On the other, inspired by an empirical study of the polynomials $A_{\lambda}^{(k)}(x ; t)$ when $t=1$, distinguished bases for $\Lambda^{(n)}$ and $\Lambda_{(n)}$ that refine the Schur basis for $\Lambda$ were introduced and connected to the quantum cohomology of Grassmannians in [LM08, LM07]. The two approaches merged when Lam proved in [Lam08] that these $k$-Schur bases are sets of representatives for the Schubert classes of $H^{*}(\mathrm{Gr})$ and $H_{*}(\mathrm{Gr})$ (where $k=n-1$ ). Moreover, the $k$-Schur functions for $\Lambda_{(k)}=\Lambda_{(k)}^{t=1}$ were conjectured [LM05] to be the parameterless $\left\{A_{\lambda}^{(k)}(x ; 1)\right\}$, suggesting a link from the theory of Macdonald polynomials to quantum and affine Schubert calculus.

Here, we circumvent the problem that the characterization for $A_{\mu}^{(k)}(x ; t)$ lacks in mechanism for proof and definitively establish this link. Our work relies on a remarkable connection between chains in the strong and the weak order poset on the type- $A$ affine Weyl group. From this, we are able to construct one parameter families of symmetric functions that transition positively with $H_{\mu}(x ; 0, t)$ and Macdonald's $P$-functions and that specialize to the Schubert representatives for 
$H^{*}(\mathrm{Gr})$ and $H_{*}(\mathrm{Gr})$ when $t=1$. The same approach leads also to a strong-cover formulation for all elements in a defining set of Gromov-Witten invariants for flag manifolds.

Our presentation begins with a fresh look at the product structure of $H^{*}(\mathrm{Gr})$ and $H_{*}(\mathrm{Gr})$. The ring is determined by multiplication of an arbitrary class with a simple class. Explicit Pieri rules for these products were given in [LM07, LLMS10]; the homology rule is framed using saturated chains in the weak order on elements in $\tilde{A}_{n-1}$ and the cohomology rule is in terms of strong order saturated chains. We distinguish a subset of these strong chains by imposing a translation and a horizontality condition on Ferrers shapes. We prove that this subset newly characterizes the homology rule, providing a cohesive framework for the structure of $H^{*}(\mathrm{Gr})$ and $H_{*}(\mathrm{Gr})$. For $w \in \tilde{S}_{n}^{0}$ and $c_{0, m}=s_{m-1} \cdots s_{1} s_{0}$,

$$
\xi_{c_{0, m}} \xi_{w}=\sum_{u \in \tilde{S}_{n}^{0}} \xi_{u}
$$

where $(\mathfrak{a}(w), \mathfrak{a}(u))$ is a horizontal strong $(n-1-m)$-strip. In essence, a horizontal strong strip is a saturated chain in the Bruhat order from $u$ to the translation of $w$ by $s_{x-1} \cdots s_{x+1}$ (see Definition 6).

From the horizontal strong strips, we derive a new combinatorial tool called affine Bruhat countertableaux (or $A B C$ 's). We prove that their generating functions are representatives for the Schubert basis of $H^{*}(\mathrm{Gr})$ and by associating a non-negative integer statistic called $n$-cocharge to each $A B C$, we refine the Kostka-Foulkes polynomials. The family of weak Kostka-Foulkes polynomials are defined, for partitions $\mu$ and $\lambda$ with parts smaller than $n$, by

$$
K_{\lambda \mu}^{n}(t)=\sum_{A \in A B C(\lambda, \mu)} t^{n \text {-cocharge }(A)}
$$

summing over all $A B C$ 's of shape $\lambda$ and weight $\mu$. A new family of symmetric functions over $\mathbb{Q}(t)$ that reduces to Schubert representatives for the cohomology of Gr when $t=1$ can then be drawn by

$$
\mathfrak{\subseteq}_{\lambda}^{(n)}(x ; t)=\sum_{\mu} K_{\lambda \mu}^{n}(t) P_{\mu}(x ; t),
$$

where $\left\{P_{\mu}(x ; t)\right\}$ are Macdonald's $P$-functions. A basis that reduces to the Schubert representatives for $H_{*}(\mathrm{Gr})$ when $t=1$ and whose transition matrix with Macdonald polynomials $H_{\mu}(x ; 0, t)$ has entries in $\mathbb{N}[t]$ is then given by the dual basis

$$
\left\{s_{\lambda}^{(n)}(x ; t)\right\},
$$

with respect to the Hall-inner product on $\Lambda$. These are conjecturally the $A_{\lambda}^{(n-1)}(x ; t)$.

Another advantage of the strongly formulated homology rule is that it allies with the combinatorial backdrop of quantum Schubert calculus. The quantum cohomology ring of the complete flag manifold $F L_{n}$ (chains of vector spaces in $\mathbb{C}^{n}$ ) decomposes into Schubert cells indexed by permutations $w \in S_{n}$. As a linear space, the quantum cohomology is $Q H^{*}\left(F L_{n}\right)=H^{*}\left(F L_{n}\right) \otimes$ $\mathbb{Z}\left[q_{1}, \ldots, q_{n-1}\right]$ for parameters $q_{1}, \ldots, q_{n-1}$ and the appeal lies in its rich multiplicative structure. The quantum product

$$
\sigma_{u} * \sigma_{v}=\sum_{w} \sum_{d} q_{1}^{d_{1}} \ldots q_{n-1}^{d_{n-1}}\langle u, v, w\rangle_{d} \sigma_{w_{0} w}
$$


is defined by 3-point Gromov-Witten invariants of genus 0 which count equivalence classes of certain rational curves in $F L_{n}$. The study of Gromov-Witten invariants is ongoing. Many attempts to gain direct combinatorial access to the structure constants have been made, but formulas are still being pursued even in the simplest case when $q_{1}=\cdots=q_{n-1}=0$. In this case, if $u$ and $v$ are permutations with one descent, the invariants reduce to the well-understood Littlewood-Richardson coefficients [LR34, MPS77]. For generic $u$ and $v$ in $S_{n}$, the invariants are the structure constants of Schubert polynomials [LS82], mysterious even when $u$ has only one descent.

Although the construction is not manifestly positive, all the Gromov-Witten invariants of (5) can be calculated from the subset

$$
\left\{\left\langle s_{r}, v, w\right\rangle_{d}: 1 \leq r<n \text { and } v, w \in S_{n}\right\} .
$$

Fomin, Gelfand, and Postnikov [FGP97] use quantum Schubert polynomials to characterize this set as a generalization of Monk's formula. Here, we approach the study by way of the affine Grassmannian. Peterson asserted that $Q H^{*}(G / P)$ of a flag variety is a quotient of the homology $H_{*}\left(\mathrm{Gr}_{G}\right)$ up to localization (detailed and proven in [LS12]). As a by-product, the three-point genus zero Gromov-Witten invariants (5) are structure constants of the Schubert basis for $H_{*}(\mathrm{Gr})$. A precise identification of $\langle u, v, w\rangle_{d}$ with coefficients $c_{\mu, \lambda}^{v}$ in

$$
\xi_{\mu} \xi_{\lambda}=\sum_{v} c_{\mu, \lambda}^{v} \xi_{v}
$$

is made in [LM] (where $\mu, v, \lambda$ are certain Ferres shapes associated to elements of $\tilde{S}_{n}^{0}$ ) and the defining set (6) of Gromov-Witten invariants is determined to be a subset of

$$
\left\{c_{R_{r}^{\prime}, \lambda}^{v}: 1 \leq r<n \text { and } \lambda, v \in C^{n}\right\}
$$

where $R_{r}^{\prime}$ is the rectangular Ferrers shape $\left(r^{n-r}\right)$ with its unique corner removed.

In this context, the set can conjecturally be characterized simply as strong covers under a rectangular translation; that is,

$$
\xi_{R_{r}^{\prime}} \xi_{\lambda}=\sum_{v \ll_{B} R(r, \lambda)} \xi_{v}
$$

where $v_{i}<R(r, \lambda)_{i}$ for some $i$ such that $\left(\lambda \cup R_{r}\right)_{i}=r$ and the $q$-parameters are readily extracted from the shape $v$. We extend the definition of horizontal strong strips to a larger distinguished subset of strong order chains characterized by a condition involving ribbon shapes (see Definition 40). The ribbon strong strips are inspired by the expansion of $\xi_{\mu} \xi_{\lambda}$, where $\mu$ is $\left(r^{n-r-1}, r-a\right)$ for $1 \leq a<r<n$.

Another motivation for this approach is in its application to an open problem in the study of $H^{*}(\mathrm{Gr})$. The problem of expanding a Schubert homology class in the affine Grassmannian of $G=S L_{n-1}$ into Schubert homology classes in Gr was settled in [LLMS12], but the cohomological picture requires a deeper understanding of the intricacies of strong strips. The ribbon strong strips point the study towards converting between weak and strong chains so that the existing work on the homology problem can be applied to the cohomology problem (see [LLMS12] for details). 


\section{Related work}

The parameterless $k$-Schur function structure constants contain all the Schubert structure constants in the quantum cohomology of flag varieties [LS12, LM]. The search for formulas for these constants is tied to many exciting projects.

The quantum comology of the Grassmannian can be accessed [BKT03] from the ordinary cohomology of two-step flags, in which case the Schubert structure constants can be computed by an iterative algorithm of Coskun [Cos09] or by Knutson-Tao puzzles [KT03] (proved in forthcoming work [BKPT, Buc]). The constants also match the structure constants of the Verlinde fusion alegbra for WZW models [Ver88, TUY89], efficiently computed by the Kac-Walton formula [Kac90, Wal90] and combinatorially attempted by [Tud00, KS10, MS12] among others.

Formulas in the quantum cohomology of flag varieties have been derived only in special cases such as the quantum Monk formula [FGP97] and quantum Pieri formula [Pos99]. These special constants were connected in [LM] to the $k$-Schur expansion of $s_{\mu}^{(k)} s_{\lambda}^{(k)}$, where $\mu$ is a rectangle minus part of a row. The $k$-Pieri rule was given in [LM07] and a more general result appears in [BBPZ12, BSS13, BSS]. The problem currently excites many perspectives including the FominKirillov algebra [MPP], the affine nil-Coxeter algebra [BBTZ12], Fomin-Greene monoids [BB96], residue tables [FK13], and crystal bases [MS].

The inclusion of a generic $t$-parameter has so far been met with limited success. Most notably, Lapointe and Pinto [PL] introduced a statistic on weak tableaux and proved that it matches the weight on the poset in [LLMS12] that describes the expansion of a Schubert homology class in Gr into Schubert homology classes in the affine Grassmannian of $G=S L(n+1, \mathbb{C})$. In [DM13], we prove that these match the statistic on $A B C$ 's. Closely related is work expressing $k$-Schur functions in terms of Kirillov-Reshetikhin crystals for type $A_{n}$ [MS].

Jing [Jin91] introduced vertex operators $B_{r}$ with the property that

$$
B_{r} H_{\mu}(x ; 0, t)=H_{r, \mu}(x ; 0, t) .
$$

These play a central role [HMZ12] in the developing theory of diagonal harmonics [GH96]. Zabrocki [Zab98] determined the action of $B_{r}$ on a Schur function, giving a new proof of the cocharge formulation for Kostka-Foulkes polynomials. In fact, our approach to the homology Pieri rule using the strong instead of the weak order came out of a study of his work and the action of $B_{r}$ on a $k$-atom

$A_{\lambda}^{(k)}(x ; t)$. A deeper understanding of the operators will shed light on open problems in diagonal harmonics and their connection to affine Schubert calculus. 


\section{Preliminaries}

The type- $A$ affine Weyl group is realized as the affine symmetric group $\tilde{S}_{n}$ given by generators $\left\{s_{0}, s_{1}, \ldots, s_{n-1}\right\}$ satisfying the relations

$$
\begin{gathered}
s_{i}^{2}=1, \\
s_{i} s_{i+1} s_{i}=s_{i+1} s_{i} s_{i+1} \\
s_{i} s_{j}=s_{j} s_{i} \quad \text { for } i-j \not \equiv 1, n-1 \quad(\bmod n)
\end{gathered}
$$

with all indices related $\bmod n$. If $w=s_{i_{1}} \cdots s_{i_{\ell}} \in \tilde{S}_{n}$ and $\ell$ is minimal among all such expressions for $w$, then $s_{i_{1}} \cdots s_{i_{\ell}}$ is called a reduced word for $w$ and the length of $w$ is defined by $\ell(w)=\ell$. The weak order on $\tilde{S}_{n}$ is defined by the covering relation

$$
w \lessdot z \Longleftrightarrow z=s_{i} w \text { and } \ell(z)=\ell(w)+1 .
$$

Alternatively, $\tilde{S}_{n}$ is the group of permutations of $\mathbb{Z}$ with the property that $w \in \tilde{S}_{n}$ acts by $w(i+r n)=w(i)+r n$, for all $r \in \mathbb{Z}$ and $\sum_{i=1}^{n} w(i)=\left(\begin{array}{c}n+1 \\ 2\end{array}\right)$. For $0 \leq i<n$, the elements $s_{i} \in \tilde{S}_{n}$ act on $\mathbb{Z}$ by $s_{i}(i+r n)=i+1+r n, s_{i}(i+1+r n)=i+r n$, and $s_{i}(j)=j$ for $j \not \equiv i, i+1(\bmod n)$.

Although the simple reflections $s_{i}$ generate the group, there is also the notion of a transposition $\tau_{i, j}$ defined by its action $\tau_{i, j}(i+r n)=j+r n$ and $\tau_{i, j}(\ell)=\ell$ for $\ell \not \equiv i, j(\bmod n)$. Take integers $i<j$ with $i \not \equiv j(\bmod n)$ and $v=\lfloor(j-i) / n\rfloor$, then $\tau_{i, i+1}=s_{i}$ and for $j-i>1$,

$$
\tau_{i, j}=s_{i} s_{i+1} s_{i+2} \cdots s_{j-v-2} s_{j-v-1} s_{j-v-2} s_{j-v-3} \cdots s_{i+1} s_{i}
$$

where the indices of simple reflections are taken $\bmod n$. For $j>i$, we set $\tau_{i, j}=\tau_{j, i}$. The strong (Bruhat) order is defined by the covering relation

$$
w \leftarrow_{B} u \quad \text { when } \quad u=\tau_{r, s} w \quad \text { and } \quad \ell(u)=\ell(w)+1 .
$$

The symmetric group $S_{n}$ can be viewed as the parabolic subgroup of $\tilde{S}_{n}$ generated by $\left\{s_{1}, s_{2}, \ldots, s_{n-1}\right\}$. The left cosets of $\tilde{S}_{n} / S_{n}$ are called affine Grassmannian elements and they are identified by the set $\tilde{S}_{n}^{0} \subset \tilde{S}_{n}$ of minimal length coset representatives. Elements of $\tilde{S}_{n}^{0}$ can be conveniently represented by a subset of partitions. A partition is a non-decreasing vector $\lambda=\left(\lambda_{1}, \ldots, \lambda_{n}\right)$ of positive integers and it is identified by its (Ferrers) shape having $\lambda_{i}$ lattice squares in the $i^{\text {th }}$ row, from the bottom to top. For partitions $\lambda$ and $\mu, \mu \subset \lambda$ when $\mu_{i} \leq \lambda_{i}$. Given $\mu \subset \lambda$, the skew shape $\lambda / \mu$ is defined by deleting the cells of $\mu$ from $\lambda$. When there is at most one cell in any column of $\lambda / \mu$, this skew shape with $m$ cells is called a horizontal $m$-strip. The cell of a horizontal 1 -strip $\lambda / \mu$ is called a removable corner of $\lambda$ and an addable corner of $\mu$. A cell $(i, j)$ of a partition $\lambda$ with $(i+1, j+1) \notin \lambda$ is called an extremal cell.

It is the subset of shapes $C^{n}$ called $n$-cores that are in bijection with affine Grassmannian permutations. An $n$-core is a partition that has no cell whose hook-length is $n$ (the hook-length of cell $c=(i, j)$ is $\lambda_{i}-i+1$ plus the number of cells in colum $j$ above cell $\left.c\right)$. The content of $c$ is $j-i$ and 
its $n$-residue is $j-i(\bmod n)$. There is a left action of the generator $s_{i} \in \tilde{S}_{n}$ on an $n$-core $\lambda$ with at least one addable corner of residue $i$; it is defined by letting $s_{i} \lambda$ be the shape where all corners of residue $i$ have been added to $\lambda$. This extends to a bijection [Las99, LM05]

$$
\mathfrak{a}: \tilde{S}_{n}^{0} \longrightarrow C^{n}
$$

where $\lambda=\mathfrak{a}(w)=s_{i_{1}} \cdots s_{i_{\ell}} \emptyset$ for any reduced word $i_{1} \cdots i_{\ell}$ of $w$. We use $w_{\lambda}$ to denote the preimage of $\lambda$ under a. From this, we define the degree of the $n$-core $\lambda$, $\operatorname{deg}(\lambda)$, to be $\ell=\ell\left(w_{\lambda}\right)$. An $n$-core $\lambda$ has an addable corner of residue $i$ if and only if

$$
\ell\left(w_{s_{i} \lambda}\right)=\ell\left(w_{\lambda}\right)+1 \text {. }
$$

Property 1. LM05] For an n-core $\lambda$ with extremal cells $c$ and $c^{\prime}$ of the same $n$-residue, given that $c$ is weakly north-west of $c^{\prime}$, if $c$ is at the end of its row, then so is $c^{\prime}$. If $c$ has a cell above it, then so does $c^{\prime}$.

The strong order on the subset $\tilde{S}_{n}^{0}$ is characterized on elements of $C^{n}$ by the containment of diagrams and its covering relation is

$$
\mu \lessdot_{B} \lambda \Longleftrightarrow \mu \subset \lambda \text { and } \operatorname{deg}(\lambda)=\operatorname{deg}(\mu)+1 .
$$

Given a pair $\mu \lessdot_{B} \lambda$, the shape $\lambda / \mu$ can be described by ribbons. For $r \geq 0$, an $r$-ribbon $R$ is a skew diagram $\lambda / \mu$ consisting of $r$ rookwise connected cells such that there is no $2 \times 2$ shape contained in $R$. In a ribbon, the southeasternmost cell is called its head and the northweasternmost cell is its tail. The height of a ribbon is the number of rows it occupies.

Lemma 2. [LLMS10] If $w \lessdot_{B} \tau_{r, s} w$ in $\tilde{S}_{n}^{0}$, then the skew $\mathfrak{a}\left(\tau_{r, s} w\right) / \mathfrak{a}(w)$ is made up of copies of one fixed ribbon such that the head of each copy has residue $s-1$ and the tail has residue $r$.

Lemma 3. Given $w \lessdot_{B} \tau_{r, s} w$ in $\tilde{S}_{n}^{0}$,

$$
\mathfrak{a}\left(\tau_{r, s} w\right)=\mathfrak{a}(w)+\text { all addable ribbons with a head of residue } r-1 \text { and tail of residue } s .
$$

Proof. Since $\tau_{r, s}=s_{r} s_{r+1} \cdots s_{s-1} \cdots s_{r}$, any addable ribbon of $\mathfrak{a}(w)$ with a head of residue $s-1$ and a tail of residue $r$ is added to $\mathfrak{a}(w)$ under multiplication by $\tau_{r, s}$. The result then follows from Lemma 2 .

\section{Affine Pieri rules}

The main discovery is that there is a fundamental connection between weak order chains from $u$ to $v$ in $\tilde{S}_{n}^{0}$ and strong order chains from $v$ to a translation of $u$. We start by addressing the case that applies to Pieri rules, in which case the translation of an element $u_{\lambda} \in \tilde{S}_{n}^{0}$ amounts to $s_{x-1} s_{x-2} \cdots s_{x+1} u_{\lambda}$ where $x=\lambda_{1}-1(\bmod n)$. 


\subsection{Strong and weak Pieri rules}

The Pieri rules for the $H^{*}(\mathrm{Gr})$ and $H_{*}(\mathrm{Gr})$ are given in [LM07, LLMS10]. The affine homology rule is framed using saturated chains in the weak order on $\tilde{S}_{n}^{0}$, whereas the cohomology rule is in terms of strong order saturated chains.

A word $a_{1} a_{2} \cdots a_{\ell}$ with letters in $\mathbb{Z} / n \mathbb{Z}$ is called cyclically decreasing if each letter occurs at most once and $i+1$ precedes $i$ whenever $i$ and $i+1$ both occur in the word. An affine permutation is called cyclically decreasing if it has a cyclically decreasing reduced word. The affine homology Pieri rule for $H_{*}(\mathrm{Gr})$ is given, for $w \in \tilde{S}_{n}^{0}$ and $c_{0, m}=s_{m-1} \cdots s_{1} s_{0} \in \tilde{S}_{n}^{0}$, by

$$
\xi_{c_{0, m}} \xi_{w}=\sum_{v} \xi_{v w}
$$

over all cyclically decreasing $v$ of length $m$ such that $v w \in \tilde{S}_{n}^{0}$ and $\ell(v w)=\ell(w)+m$. Thus, for $u \in \tilde{S}_{n}^{0}$, the term $\xi_{u}$ occurs in the product of $\xi_{c_{0, m}} \xi_{w}$ only when $u w^{-1}$ is cyclically decreasing and there is a saturated chain

$$
w=w^{(0)} \lessdot w^{(1)} \lessdot \cdots \lessdot w^{(m)}=u .
$$

Alternatively, the rule can be formulated in the language of shapes using the action of the $s_{i}{ }^{-}$ generators on $n$-cores.

Lemma 4. [LLMS10] For $u, w \in \tilde{S}_{n}^{0}$ where $\ell\left(u w^{-1}\right)=m, u w^{-1}$ is cyclically decreasing with reduced word $j_{1} \cdots j_{m}$ if and only if $\mathfrak{a}(u) / \mathfrak{a}(w)$ is a horizontal strip such that the set of residues labelling its cells is $\left\{j_{1}, \ldots, j_{m}\right\}$.

While the weak order determines the affine homology rule for $H_{*}(\mathrm{Gr})$, the affine cohomology Pieri rule is given as the sum over certain multisets of chains in the Bruhat (strong) order. The multisets arise by imposing a marking on strong covers $\rho \lessdot_{B} \gamma$ in $C^{n}$. Define $(\gamma, c)$ to be a marked strong cover of $\rho$ if $\rho \leftarrow_{B} \gamma$ and $c$ is the content of the head of a ribbon in $\gamma / \rho$ (recall that Lemma2 assures the skew shape is made up of ribbons). Then, for $0 \leq m<n$ and $n$-cores $v$ and $\gamma$, a strong $m$-strip from $v$ to $\gamma$ is a saturated chain of cores

$$
v=\gamma^{(0)} \lessdot_{B} \gamma^{(1)} \lessdot_{B} \ldots \lessdot_{B} \gamma^{(m)}=\gamma,
$$

together with an increasing "content vector" $c=\left(c_{1}, c_{2}, \cdots, c_{m}\right)$, such that $\left(\gamma^{(i)}, c_{i}\right)$ is a marked strong cover of $\gamma^{(i-1)}$ for $1 \leq i \leq m$.

Example 5. For $n=4$, there are 2 saturated chains from $v=(3)$ to $\gamma=(4,1,1)$,

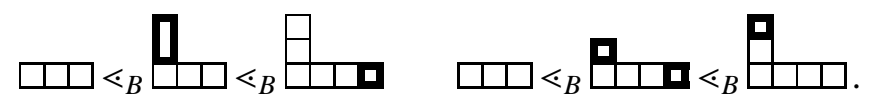

The first chain with content vector $c=(-1,3)$ is thus the only strong 2-strip from $v$ to $\gamma$. 
The affine cohomology Pieri rule is

$$
\xi^{c, m} \xi^{w}=\sum_{S} \xi^{z}
$$

where the sum runs over strong $m$-strips $S$ from $\mathfrak{a}(w)$ to $\mathfrak{a}(z)$. In contrast to the Pieri rule for $H_{*}(\mathrm{Gr})$, a given term $\xi^{z}$ here may occur with multiplicity greater than 1 .

\subsection{Horizontal strong strips}

As with the affine Pieri rule for the cohomology $H^{*}(\mathrm{Gr})$, the Pieri rule [BS98] and the quantum Pieri rule [FGP97] for (quantum) cohomology of the flag manifold are also determined by chains in the Bruhat order (see (32)). However, it is the homology of Gr, not the cohomology, that is algebraically tied to the quantum cohomology of the flag manifold (detailed in $\S$ 7). To align the combinatorics with the algebra, we introduce a distinguished subclass of strong order chains that characterize the affine homology Pieri rule. The fundamental observation is that the translation of an $n$-core $\lambda$ to the $n$-core $R(n-1, \lambda)=\left(\lambda_{1}+n-1, \lambda\right)$ plays a crucial role.

Definition 6. A pair of $n$-cores $(\lambda, v)$ is a horizontal strong $m$-strip if $\lambda \subset v$ and there is a saturated chain of cores

$$
v=v^{(0)} \lessdot_{B} v^{(1)} \lessdot_{B} \cdots \lessdot_{B} v^{(m)}=R(n-1, \lambda)
$$

such that the bottom row of $v^{(i)}$ is longer than the bottom row of $v^{(i-1)}$, for $1 \leq i \leq m$ where $m=n-1+\operatorname{deg}(\lambda)-\operatorname{deg}(v)$.

Example 7. For $n=4, \lambda=(1,1)$, and $v=(3),(\lambda, v)$ is not a horizontal strong strip since neither of the strong chains from $v$ to $R(n-1, \lambda)$ shown in (11) have strictly growing bottom rows.

For $\lambda=(3,1,1)$, the 4-cores $v$ such that $(\lambda, v)$ is a horizontal strong 2-strip begin with the chains to $R(3, \lambda)$ :

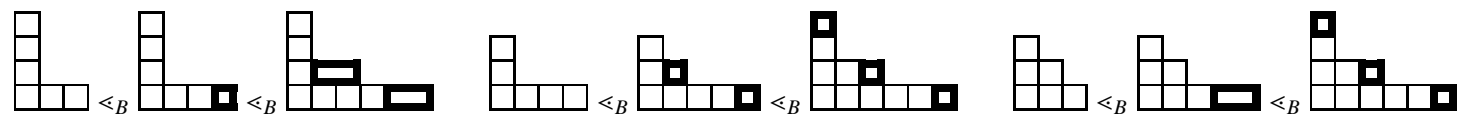

We have chosen the terminology horizontal strong strip because, although not immediately obvious, there always exists a strong strip from $v$ to $R(n-1, \lambda)$ of shapes that differ by ribbons of height one when $(\lambda, v)$ is a horizontal strong strip. The following lemma associates horizontal strong strips to the horizontality condition and we then connect to strong strips.

Lemma 8. Given $n$-cores $\lambda \subset v$ and a saturated chain of shapes (13) whose bottom rows strictly increase, there are adjacent ribbons $S^{1}, \ldots, S^{m}$ in the bottom row of $R(n-1, \lambda) / \lambda$ such that the shape $v^{(j)} / v^{(j-1)}$ is comprised of all copies of $S^{j}$ that can be removed from $v^{(j)}$, for each $1 \leq j \leq m$.

Proof. Consider $\lambda \subset v$ and a chain of $n$-cores (13) where the bottom rows increase in size. Let $S^{j}$ denote the lowest ribbon in $v^{(j)} / v^{(j-1)}$. Since the bottom row of $v^{(j)}$ is strictly longer than the 
bottom of $v^{(j-1)}$, the head of $S^{j}$ lies in the bottom row of $v^{(j)}$. Moreover, $S^{j}$ has height one since $\lambda \subset v^{(j)} \subset\left(n-1+\lambda_{1}, \lambda\right)$ and $\left(n-1+\lambda_{1}, \lambda\right) / \lambda$ is a horizontal strip. Therefore, $S^{j}$ is a removable ribbon lying entirely in the bottom row of $v^{(j)}$. Lemma 3 then implies that $v^{(j)} / v^{(j-1)}$ consists of all copies of $S^{j}$ that can be removed from $v^{(j)}$.

Proposition 9. For $n$-cores $\lambda \subset v$, the pair $(\lambda, v)$ is a horizontal strong $m$-strip if and only if there exists a strong $m$-strip from $v$ to $R(n-1, \lambda)$ whose content vector $c$ satisfies $c_{1} \geq \lambda_{1}$.

Proof. Given any horizontal strong $m$-strip $(\lambda, v)$, we have a chain (13) that is characterized by ribbons $S^{1}, \ldots, S^{m}$ lying in the bottom row of $R(n-1, \lambda) / v$ by Lemma 8 . We can obtain a strong strip by associating it to the content vector $\left(c_{1}, \ldots, c_{m}\right)$, where $c_{i}$ is the content of the head of ribbon $S^{i}$. Then $c_{1} \geq \lambda_{1}$ since $\lambda \subset v$.

On the other hand, consider cores $v=v^{(0)} \lessdot_{B} v^{(1)} \lessdot_{B} \cdots \lessdot_{B} v^{(m)}=R(n-1, \lambda)$ such that the head $h_{i}$ of a ribbon in $v^{(i)} / v^{(i-1)}$ has content $c_{i}$ and $\lambda_{1} \leq c_{1}<\cdots<c_{m}$. The last $n-1$ cells in the bottom row of $R(n-1, \lambda)$ lie at the top of their column and therefore they are the only cells with content greater than $\lambda_{1}-1$. Therefore, $h_{i}$ must lie in the bottom row of $v^{(i)} \subset R(n-1, \lambda)$ implying that bottom rows are strictly growing.

Remark 10. The proof of Theorem 14 will establish a claim stronger than Proposition 9. each horizontal strong strip $(\lambda, v)$ corresponds uniquely to a strong strip from $v$ to $R(n-1, \lambda)$ with $c_{1} \geq \lambda_{1}$.

Example 11. For $n=4$ and $\lambda=(3,1,1)$, the $n$-cores $v$ such that $(\lambda, v)$ is a horizontal strong 2-strip are given in Example 7 and each corresponds to a unique strong 2-strip from $v$ to $R(3, \lambda)$ with $c_{1} \geq 3$ : their content vectors are $(3,5),(4,5)$, and $(4,5)$, respectively.

Horizontal strong strips in hand, we now discuss their correspondence with weak order cyclically decreasing chains. For a fixed $x \in[n]=\{0, \ldots, n-1\}$ and $y \in\{0, \ldots, n-1\} \backslash\{x\}$, it will be inferred that $x+1 \leq y \leq x-1$ is taken with respect to the total order defined by

$$
x+1<x+2<\cdots<0<n-1<\cdots<x-1 .
$$

For $n$-cores $\lambda$ and $v$, a simple construction produces a cyclically decreasing word for $w_{v} w_{\lambda}^{-1}$ from a relevant strong chain from $v$ to $R(n-1, \lambda)$. For $x=\lambda_{1}-1(\bmod n)$, define the map

$$
\psi: v=v^{(0)} \lessdot_{B} v^{(1)} \lessdot_{B} \cdots \lessdot_{B} v^{(m)}=R(n-1, \lambda) \quad \longmapsto \quad s_{j_{1}} \cdots s_{j_{n-1-m}}
$$

where the elements $j_{1}>\cdots>j_{n-1-m}$ of $\{x-1, \ldots, x+1\} \backslash\left\{a_{1}, \ldots, a_{m}\right\}$ are obtained by taking $a_{m-i}$ to be the residue of the leftmost cell in the bottom row of $v^{(i+1)} / v^{(i)}$, for $0 \leq i<m$. In reverse, a strong chain arises from a reduced expression for $w_{\nu} w_{\lambda}^{-1}$ with the map

$$
\phi: s_{j_{1}} \cdots s_{j_{n-1-m}} \longmapsto v=v^{(0)} \subset v^{(1)} \subset \cdots \subset v^{(m)}=R(n-1, \lambda),
$$


where $v^{(i)}$ is obtained from $v^{(i+1)}$ by deleting all removable copies of the ribbon whose tail has residue $a_{m-i}$ and lies in the bottom row, where $x+1 \leq a_{m}<\cdots<a_{1} \leq x-1$ are the elements of $\{x-1, \ldots, x+1\} \backslash\left\{j_{1}, \ldots, j_{n-1-m}\right\}$.

Several lemmas are first needed to prove that $\phi$ and $\psi$ give the desired bijection. Horizontal strong strips $(\lambda, v)$ are defined on the level of cores where the key idea is to study strong chains from $v$ to the $n$-translation of $\lambda$ defined by $R(n-1, \lambda)$. A preliminary result puts the idea of this translation into the framework of the affine Weyl group.

Lemma 12. For $w_{\lambda} \in \tilde{S}_{n}^{0}$, the length $\ell\left(w_{R(n-1, \lambda)}\right)=n-1+\ell\left(w_{\lambda}\right)$ and

$$
w_{R(n-1, \lambda)}=s_{x-1} \cdots s_{x+1} w_{\lambda},
$$

where $x=\lambda_{1}-1(\bmod n)$.

Proof. It suffices to prove that $R(n-1, \lambda)=\mathfrak{a}\left(s_{x-1} \cdots s_{x+1} w\right)$. Since the lowest addable corner of $\lambda$ has residue $x+1, s_{x+1}$ acts on $\lambda$ by adding all corners of residue $x+1$. Similarly, $s_{x+2}$ adds corners of residue $x+2$ and by iteration, the degree of $\lambda$ increases by $n-1$ under the action of $s_{x-1} \cdots s_{x+1}$. Since $s_{x-1} \cdots s_{x+1}$ is cyclically decreasing, Lemma 4 implies that it acts on $\lambda$ by adding a horizontal strip. The result follows by noting that $R(n-1, \lambda)$ is the unique core obtained by adding a horizontal strip to $\lambda$ and increasing degree by $n-1$.

For $x \in\{0,1, \ldots, n-1\}$, let $S_{\hat{x}}=\left\langle s_{0}, \ldots, \hat{s}_{x}, \ldots, s_{n-1}\right\rangle \subset \tilde{S}_{n}^{0}$ be the subgroup generated by all simple reflections except $s_{x}$.

Lemma 13. Given $w_{\lambda}, u \in \tilde{S}_{n}^{0}$ where $u w_{\lambda}^{-1}$ is a cyclically decreasing permutation and $\ell\left(u w_{\lambda}^{-1}\right)=$ $\ell(u)-\ell\left(w_{\lambda}\right)$, then $u w_{\lambda}^{-1} \in S_{\hat{x}}$ for $x=\lambda_{1}-1(\bmod n)$.

Proof. Let $v=s_{j_{1}} \cdots s_{j_{m}}$ be a reduced expression for $u w_{\lambda}^{-1}$. By the definition of $\mathfrak{a}$, the residues labelling the cells in $D=\mathfrak{a}\left(v w_{\lambda}\right) / \lambda$ come from the set $\left\{j_{1}, \ldots, j_{m}\right\}$. In fact, since $\ell\left(v w_{\lambda}\right)=\ell\left(w_{\lambda}\right)+m$, the cells of $D$ are labelled by precisely the set $\left\{j_{1}, \ldots, j_{m}\right\}$. Since $v$ is cyclically decreasing, we also have that $D$ is a horizontal strip by Lemma 4. Therefore, an extremal cell of residue $j_{t}$ that does not lie at the end of its row occurs in $\lambda$ for every $1 \leq t \leq m$. Since $x$ is the residue of the last cell in the bottom row of $\lambda$, Property 1 implies that every extremal cell of $\lambda$ with residue $x$ lies at the end of its row. In particular, $x \neq j_{t}$ and we have $v \in S_{\hat{x}}$.

Theorem 14. For $n$-cores $\lambda$ and $v,(\lambda, v)$ is a horizontal strong strip if and only if $w_{v} w_{\lambda}^{-1}$ is a cyclically decreasing permutation where $\ell\left(w_{v}\right)=\ell\left(w_{\lambda}\right)+\ell\left(w_{v} w_{\lambda}^{-1}\right)$.

Proof. From a horizontal strong $m$-strip $(\lambda, v)$, Lemma 8 gaurantees us a chain $v=v^{(0)} \lessdot_{B} v^{(1)} \lessdot_{B}$ $\cdots \lessdot_{B} v^{(m)}=R(n-1, \lambda)$, such that a ribbon $S^{i+1}$ of $v^{(i+1)} / v^{(i)}$ has height one and is a removable ribbon in the bottom row of $v^{(i+1)}$. It suffices to prove that the image $s_{j_{1}} \cdots s_{j_{t}}$ of this chain under $\psi$ is a cyclically decreasing word for $w_{v} w_{\lambda}^{-1}$ of length $n-1-m$ since the definition of horizontal $m$-strip implies that $\ell\left(w_{v}\right)=n-1-m+\ell\left(w_{\lambda}\right)$. 
The definition of $\psi$ uses $a_{m-i}$ to denote the residue of the tail of $S^{i+1}$ and thus the residue of the head of $S^{i}$ must be $a_{m-i}-1$. By Lemma 2, we have $w_{v^{(i)}}=\tau_{a_{m-i}, a_{m-i-1}} w_{v^{(i+1)}}$ for $0 \leq i<m$, where $a_{0}=\lambda_{1}-1(\bmod n)$. In particular, $w_{v}=\tau_{a_{m}, a_{m-1}} \cdots \tau_{a_{1}, a_{0}} w_{R(n-1, \lambda)}$. Since $\lambda \subset v$, we have that $\lambda_{1} \leq a_{m}$ and therefore $x+1 \leq a_{m}<\cdots<a_{1} \leq x-1$ for $x=\lambda_{1}-1(\bmod n)$. It follows from Lemma 12 that

$$
w_{v} w_{\lambda}^{-1}=\tau_{a_{m}, a_{m-1}} \cdots \tau_{a_{1}, x}\left(s_{x-1} \cdots s_{x+1}\right),
$$

or $w_{v} w_{\lambda}^{-1}=s_{j_{1}} \cdots s_{j_{n-1-m}}$ where $j_{1}>\cdots>j_{n-1-m}$ are the elements of $\{x-1, \ldots, x+1\} \backslash\left\{a_{1}, \ldots, a_{m}\right\}$. Since these are $n-1-m$ distinct elements, the expression is reduced.

Before proving the reverse direction, note that $s_{j_{1}} \cdots s_{j_{n-1-m}}$ is the unique reduced expression for $w_{v} w_{\lambda}^{-1}$ that is ordered by $x-1 \geq j_{1}>\cdots>j_{n-1-m} \geq x+1$ and it is determined uniquely from ribbon tails in the given chain. Since a given chain under consideration is determined uniquely by its ribbon tails, the uniqueness claim of Remark 10 follows.

Suppose now that $j_{1} \cdots j_{n-1-m}$ is a reduced word for a cyclically decreasing permutation $w_{v} w_{\lambda}^{-1}$ where $\ell\left(w_{v}\right)=\ell\left(w_{\lambda}\right)+n-1-m$. By Lemma 13, $w_{\nu} w_{\lambda}^{-1} \in S_{\hat{x}}$ for $x=\lambda_{1}-1(\bmod n)$ and therefore there are $m$ elements $x-1 \geq a_{1}>a_{2}>\cdots>a_{m} \geq x+1$ in the set $\{x-1, \ldots, x+1\} /\left\{j_{1}, \ldots, j_{n-1-m}\right\}$. The $n-1$ removable cells in the bottom row of $R(n-1, \lambda)$, of residues $x-1, \ldots, x+1$ from right to left, can thus be tiled uniquely into ribbons whose tails are $a_{1}, \ldots, a_{m}$, from right to left. Therefore, the shapes in the image of $j_{1} \cdots j_{n-1-m}$ under $\phi$

$$
v=v^{(0)} \subset v^{(1)} \subset \cdots \subset v^{(m)}=R(n-1, \lambda)
$$

have increasing bottom rows. We claim this is a strong saturated chain and $\lambda \subset v$.

Let $\eta^{(m)}=v^{(m)}$ so that by Lemma12, $w_{\eta^{(m)}}=\left(s_{x-1} \cdots s_{x+1}\right) w_{\lambda}$. For $1 \leq i \leq m$, define

$$
w_{\eta^{(m-i)}}=\tau_{a_{i}, a_{i-1}} w_{\eta^{(m-i+1)}}=\left(s_{x-1} \cdots \hat{s}_{a_{1}} \cdots \hat{s}_{a_{2}} \cdots \cdots \hat{s}_{a_{i}} \cdots s_{x+1}\right) w_{\lambda},
$$

where $a_{0}=x$. Since $w_{\eta^{(0)}}=s_{j_{1}} \cdots s_{j_{n-1-m}} w_{\lambda}$, we have that $\lambda \subset \eta^{(0)}$ by Lemma4 If $w_{\eta^{(m-i)}}{ }_{B} w_{\eta^{(m-i+1)}}$, then $\eta^{(m-i)}=v^{(m-i)}$ by Lemma 3 and the claim follows. To ensure that $w_{\eta^{(m-i)}} \ll_{B} w_{\eta^{(m-i+1)}}$, it suffices to show that $w_{\eta^{(m-i+1)}}$ has length $n-i+\ell\left(w_{\lambda}\right)$. Note that $\ell\left(w_{\eta^{(0)}}\right)=n-1-m+\ell\left(w_{\lambda}\right)$ and consider $w_{\eta^{(m-i)}}$ of length $n-1-i+\ell\left(w_{\lambda}\right)$. By commuting relations, $w_{\eta^{(m-i)}}=\left(\hat{s}_{a_{i-1}} \cdots \hat{s}_{a_{i}}\right) w_{\mu}$ for $w_{\mu}=\left(s_{x-1} \cdots \hat{s}_{a_{1}} \cdots \cdots \hat{s}_{a_{i-1}}\right)\left(\hat{s}_{a_{i}} \cdots s_{x+1}\right) w_{\lambda}$. Since the lowest addable corner of $\lambda$ has residue $x+1$, the lowest addable corner of $\mu$ has residue $a_{i}$. Therefore, $w_{\eta^{(m-i+1)}}=\left(\hat{s}_{a_{i-1}} \cdots s_{a_{i}+1} s_{a_{i}}\right) w_{\mu}$ has length $n-i+\ell\left(w_{\lambda}\right)$.

Corollary 15. For $1 \leq m<n$ and $w \in \tilde{S}_{n}^{0}$,

$$
\xi_{c_{0, m}} \xi_{w}=\sum_{u \in \tilde{S}_{n}^{0}} \xi_{u}
$$

where the sum is over $u$ such that $(\mathfrak{a}(w), \mathfrak{a}(u))$ is a horizontal strong $(n-1-m)$-strip.

Proof. A term $v=w_{v} w_{\lambda}^{-1}$ occurs in the summand of (10) if and only if it is cyclically decreasing of length $m$ and $\ell\left(w_{v}\right)=\ell\left(w_{\lambda}\right)+m$. That is, if and only if $(\lambda, v)$ is a horizontal strong $n-1-m$-strip by Theorem 14 . 
Example 16. The expansion $\xi_{c_{0,2}} \xi_{(3,1,1)}=\xi_{(3,1,1,1)}+\xi_{(4,1,1)}+\xi_{(3,2,1)}$ follows from Example 7 and Corollary 15

\section{Explicit representatives for Schubert classes}

The perspective of horizontal strong strips applies to the study of the (co)homology classes of the affine Grassmannian. Here we derive a new combinatorial object with which to study the representatives for Schubert classes of $H^{*}(\mathrm{Gr})$ and $H_{*}(\mathrm{Gr})$.

\subsection{Polynomial realization of $H^{*}(\mathbf{G r})$ and $H_{*}(\mathbf{G r})$}

Quillen (unpublished) and Garland and Raghunathan [GR75] showed that Gr is homotopy-equivalent to the group $\Omega S U(n, \mathbb{C})$ of based loops into $S U(n, \mathbb{C})$. Results from [Bot58] can be used to obtain a polynomial identification of $H^{*}(\mathrm{Gr})$ and $H_{*}(\mathrm{Gr})$ inside the ring of symmetric functions $\Lambda=\mathbb{Z}\left[h_{1}, h_{2}, \ldots,\right]$, where $h_{r}=\sum_{1 \leq i_{1} \leq \cdots \leq i_{r}} x_{i_{1}} \cdots x_{i_{r}}$.

Traditionally, bases for the space of symmetric function are indexed by partitions. Descriptions of the homology and cohomology ring are most natural in terms of the functions defined by setting $h_{\lambda}=h_{\lambda_{1}} \cdots h_{\lambda_{\ell}}$ and the monomial symmetric functions $m_{\lambda}$, defined for each partition $\lambda$ as the sum over $x^{\alpha}$ for each distinct rearrangement $\alpha$ of the parts of $\lambda$. The homology $H_{*}(\mathrm{Gr})$ is identified by the subring $\Lambda_{(n)}$ of $\Lambda$ and the cohomology $H^{*}(\mathrm{Gr})$ can be identified by the quotient $\Lambda^{(n)}$ where

$$
\Lambda_{(n)}=\mathbb{Z}\left[h_{1}, \ldots, h_{n-1}\right] \quad \text { and } \quad \Lambda^{(n)}=\Lambda /\left\langle m_{\lambda}: \lambda_{1} \geq n\right\rangle .
$$

These spaces are naturally paired under the Hall-inner product on $\Lambda$, defined by setting

$$
\left\langle h_{\lambda}, m_{\mu}\right\rangle=\delta_{\lambda \mu}
$$

where $\delta_{\lambda \mu}=0$ when $\lambda \neq \mu$ and is 1 otherwise.

The Schur function basis for $\Lambda$ is self-dual with respect to $\langle$,$\rangle . Recall that this basis is a$ fundamental combinatorial tool to study tensor products of irreducible representations and intersections in the geomety of the Grassmannian variety. Schur functions are the generating functions of tableaux. A semi-standard tableau of weight $\mu=\left(\mu_{1}, \ldots, \mu_{r}\right)$ is a nested sequence of partitions

$$
\emptyset=\lambda^{(0)} \subset \lambda^{(1)} \subset \cdots \subset \lambda^{(r)}
$$

such that $\lambda^{(i)} / \lambda^{(i-1)}$ is a horizontal $\mu_{i}$-strip. It is generally represented with a filling of shape $\lambda^{(r)}$ by placing $i$ in the cells of the skew $\lambda^{(i)} / \lambda^{(i-1)}$. When the weight of a tableau is $(1,1, \ldots, 1)$ it is called standard. SS ST $(\lambda, \mu)$ denotes the set of semi-standard tableaux of shape $\lambda$ and weight $\mu$ and the union over all weights is $S S Y T(\lambda)$. For any partition $\lambda$, the Schur function is

$$
s_{\lambda}=\sum_{T \in S S Y T(\lambda)} x^{\operatorname{weight}(T)} .
$$


Refinements of the Schur basis for $\Lambda$ to bases for $\Lambda^{(n)}$ and $\Lambda_{(n)}$ give a combinatorial framework that can be applied to the cohomology and homology of Gr. Let $k=n-1$ throughout. The basis of $k$-Schur functions for $\Lambda_{(n)}$ was introduced in [LM05], inspired by the Macdonald polynomial study of [LLM03] summarized in the introduction. The basis for $\Lambda^{(n)}$ that is dual to the $k$-Schur basis with respect to the Hall-inner product arose in the context of the quantum cohomology of Grassmannians in [LM08]. Appealing to the algebraic nil-Hecke ring construction of Kostant and Kumar [KK86] and the work of Peterson [Pet97], Lam [Lam08] proved that the Schubert classes $\xi_{w}$ and $\xi^{w}$ can be represented explicitly by the $k$-Schur functions in $\Lambda_{(n)}$ and $\Lambda^{(n)}$, respectively.

For our purposes, we define the $k$-Schur functions of $H^{*}(\mathrm{Gr})$ as the weight generating functions of a combinatorial object called affine factorizations and then introduce the homology $k$-Schur functions by duality. For any composition $\alpha \in \mathbb{N}^{\ell}$ with parts smaller than $n$ and $w \in \tilde{S}_{n}$ of length $|\alpha|$, an affine factorization for $w$ of weight $\alpha$ is a decomposition

$$
w=v^{\ell} \cdots v^{1}
$$

where $v^{i}$ is a cyclically decreasing permutation of length $\alpha_{i}$. The representatives for the Schubert classes of $H^{*}(\mathrm{Gr})$ are then defined, for $\lambda \in C^{n}$, by

$$
\Im_{\lambda}^{(n)}=\sum_{w_{\lambda}=v^{r} \cdots v^{1}} x_{1}^{\ell\left(v^{1}\right)} \cdots x_{r}^{\ell\left(v^{r}\right)}
$$

over all affine factorizations $v^{r} \cdots v^{1}$ of $w$ (In [Lam06], by dropping the condition that $w_{\lambda}$ is affine Grassmannian, these are extended to a more general family of functions that relate to the stable limits of Schubert polynomials [LS82, Sta84]). The set $\left\{\mathfrak{S}_{\lambda}^{(n)}\right\}_{\lambda \in C^{n}}$ is a basis for $\Lambda^{(n)}$ and we take the $k$-Schur representatives for Schubert classes of $H_{*}(\mathrm{Gr})$ to be the dual basis $\left\{s_{v}^{(n)}\right\}_{v \in C^{n}}$ with respect to the Hall-inner product. That is, the $k$-Schur functions are defined by the relation

$$
\left\langle\mathfrak{S}_{\lambda}^{(n)}, s_{v}^{(n)}\right\rangle=\delta_{\lambda v} .
$$

\subsection{Affine Bruhat countertableaux}

Here we derive a new combinatorial object with which to study $H^{*}(\mathrm{Gr})$ and $H_{*}(\mathrm{Gr})$ by considering the association between cyclically decreasing permutations and horizontal strong strips that was made in Section 4.2. Recall that the sequence (15) can be represented by its countertableau filling, derived by placing an $r+1-i$ in $\lambda^{(i)} / \lambda^{(i-1)}$.

Definition 17. Fix composition $\alpha=\left(\alpha_{1}, \ldots, \alpha_{r}\right)$ with $\alpha_{i}<n$ and $n$-core $\lambda^{(r)}$ of degree $|\alpha|$. An affine Bruhat countertableau of shape $\lambda^{(r)}$ and weight $\alpha$ is a skew tableau $\lambda^{(r)}=\mu^{(0)} \subset \cdots \subset \mu^{(r)}$ such that

$$
\mu^{(x)}=\left(\mu_{1}^{(1)}, \ldots, \mu_{x-1}^{(x-1)}, \lambda_{1}^{(r-x)}+n-1, \lambda^{(r-x)}\right),
$$

where $\left(\lambda^{(x-1)}, \lambda^{(x)}\right)$ is a horizontal strong $\left(n-1-\alpha_{x}\right)$-strip for $1 \leq x \leq r$ and $\lambda^{(0)}=\emptyset$. 
An affine Bruhat countertableau (or $A B C$ ) is represented by its skew countertableau filling where $r-x+1$ is placed in the cells of $\mu^{(x)} / \mu^{(x-1)}$. We denote the set of $A B C$ 's of shape $\lambda$ and weight $\alpha$ by $A B C(\lambda, \alpha)$ and let $A B C(\lambda)$ be their union over all weights $\alpha$.

Example 18. For $n=6, \mu^{(0)}=(4,3,0) \subset(9,4,2) \subset(9,8,3) \subset(9,8,5)=\mu^{(3)}$ is an ABC of shape $(4,3)$ and weight $(3,3,1)$, represented by its countertableau filling

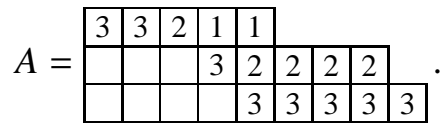

The corresponding strong strips are

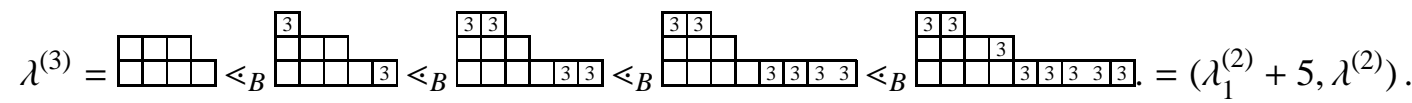

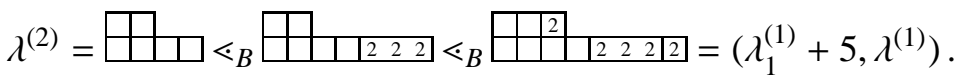

$$
\begin{aligned}
& \lambda^{(1)}=\square \square \lessdot_{B} \square \square \square \lessdot_{B} \square \square \square 11=\left(\lambda^{(0)}+5\right) .
\end{aligned}
$$

Lemma 19. Relation (18) uniquely identifies the element $\mu^{(0)} \subset \cdots \subset \mu^{(r)}$ in $A B C\left(\mu^{(0)}, \alpha\right)$ with the sequence $\emptyset=\lambda^{(0)} \subset \lambda^{(1)} \subset \cdots \subset \lambda^{(r)}=\mu^{(0)}$ where $\left(\lambda^{(p-1)}, \lambda^{(p)}\right)$ is a horizontal strong $\left(n-1-\alpha_{p}\right)$ strip,

Proof. The forward direction is immediate from the definition of $A B C$. On the other hand, consider a sequence $\emptyset=\lambda^{(0)} \subset \lambda^{(1)} \subset \cdots \subset \lambda^{(r)}$ where $\left(\lambda^{(p-1)}, \lambda^{(p)}\right)$ is a horizontal strong $\left(n-1-\alpha_{p}\right)$-strip for $1 \leq p \leq r$. Let $\mu^{(0)}=\lambda^{(r)}$, and for $1 \leq p \leq r$, define $\mu^{(p)}$ by Relation (18). From this, we find that

$$
\mu^{(p)}=\left(\lambda_{1}^{(r-1)}+n-1, \ldots, \lambda_{1}^{(r-p+1)}+n-1, R\left(n-1, \lambda^{(r-p)}\right)\right),
$$

and need only to confirm that $\mu^{(p)} / \mu^{(p-1)}$ is a horizontal strip for $1 \leq p \leq r$. Note that

$$
\mu^{(p-1)}=\left(\lambda_{1}^{(r-1)}+n-1, \ldots, \lambda_{1}^{(r-p+1)}+n-1, \lambda^{(r-p+1)}\right) .
$$

The claim follows by recalling that when $\left(\lambda^{(r-p)}, \lambda^{(r-p+1)}\right)$ is a horizontal strong strip, $R\left(n-1, \lambda^{(r-p)}\right) / \lambda^{(r-p+1)}$ is a horizontal strip.

Theorem 20. For any $n$-core $\lambda$,

$$
\Xi_{\lambda}^{(n)}=\sum_{A \in A B C(\lambda)} x^{\text {weight }(A)}
$$

Proof. Fix a composition $\alpha$ of length $r$ with parts smaller than $n$ and an $n$-core $\lambda$ such that $\operatorname{deg}(\lambda)=$ $|\alpha|$. Define a map on domain $A B C(\lambda, \alpha)$ by sending

$$
\Theta: A \longmapsto v^{r} \cdots v^{1} \quad \text { where } v^{i}=w_{\lambda^{(i)}} w_{\lambda^{(i-1)}}^{-1},
$$


for the unique sequence $\lambda^{(0)} \subset \cdots \subset \lambda^{(r)}$ associated to $A$ via Lemma 19. We claim that $\Theta$ is a bijection whose image is the set of affine factorizations of $w_{\lambda}$ of weight $\alpha$. For this, we must prove that $v^{i}$ is a cyclically decreasing permutation of length $\alpha_{i}, \ell\left(w_{\lambda}\right)=|\alpha|$ and that $\Theta$ is a bijection.

When $\alpha=\left(\alpha_{1}\right)$ has only one part, the unique element of $A B C(\lambda, \alpha)$ corresponds to the sequence $\emptyset \subset \lambda$ where $\lambda=\left(\alpha_{1}\right)$. Its image under $\Theta$ is $v^{1}=w_{\left(\alpha_{1}\right)}=s_{\alpha_{1}-1} \cdots s_{0}$, the only decomposition of $w_{\lambda}$ into one cyclically decreasing permutation $v^{1}$ of length $\alpha_{1}$. By induction, assume that the sequence $\emptyset=\lambda^{(0)} \subset \cdots \subset \lambda^{(r-1)}$, where $\left(\lambda^{(j-1)}, \lambda^{(j)}\right)$ is a horizontal strong $\left(n-1-\alpha_{j}\right)$-strip, corresponds uniquely to a decomposition of $w_{\lambda^{(r-1)}}=v^{r-1} \cdots v^{1}$ into cyclically decreasing permutations $v^{j}$ of length $\alpha_{j}$, for $j<r$. Since $v^{r}=w_{\lambda^{(r)}} w_{\lambda^{(r-1)}}^{-1}$ is cyclically decreasing of length $\alpha_{r}$ if and only if $\left(\lambda^{(r-1)}, \lambda^{(r)}\right)$ is a horizontal strong $\left(n-1-\alpha_{r}\right)$-strip by Theorem 14, the result follows by induction.

Because $\mathfrak{S}_{\lambda}^{(n)}$ is a symmetric function, the coefficient of $x^{\alpha}$ in (19) equals the coefficient of $m_{\mu}$, where $\mu$ is the non-increasing rearrangement of the parts of $\alpha$. The set of monomial symmetric functions indexed by elements in $\mathcal{P}^{n}=\left\{\lambda \in \mathcal{P}: \lambda_{1}<n\right\}$ is a basis for $\Lambda^{(n)}$ and in fact, the transition matrix from $\left\{\Im_{\lambda}^{(n)}\right\}_{\lambda \in C^{n}}$ to $\left\{m_{\mu}\right\}_{\mu \in \mathcal{P}^{n}}$ is unitriangular. The unitriangularity relation is described by an identification from [LM05] of $n$-cores with partitions of $\mathcal{P}^{n}$, given by the map

$$
\mathfrak{c}: \mathcal{P}^{n} \longrightarrow C^{n}
$$

where $\mathfrak{c}^{-1}(\gamma)=\left(\lambda_{1}, \ldots, \lambda_{\ell}\right)$ and $\lambda_{i}$ is the number of cells in row $i$ of $\gamma$ with hook-length smaller than $n$. The unitriangularity relation is taken with respect to the dominance order on partitions of the same degree, where $\lambda \triangleleft \mu$ when $\lambda_{1}+\cdots+\lambda_{s} \leq \mu_{1}+\cdots+\mu_{s}$ for all $s$. It was proven in [LM05, Lam06] that for any $\lambda \in \mathcal{P}^{n}$,

$$
\Im_{c(\lambda)}^{(n)}=m_{\lambda}+\sum_{\substack{\mu \in \mathcal{P}^{n} \\ \mu \bowtie \lambda}} K_{\lambda \mu}^{n} m_{\mu} .
$$

Corollary 21. For $\lambda, \mu \in \mathcal{P}^{n}, K_{\lambda \mu}^{n}$ is the number of $A B C$ 's of shape $c(\lambda)$ and weight $\mu$. In particular, there is a unique $A B C$ of shape $\mathrm{c}(\lambda)$ and weight $\lambda$ and $A B C(\mathfrak{c}(\lambda), \mu)=\emptyset$ when $\mu \Varangle \lambda$.

Proposition 22. [LM07] For an n-core $\lambda$ where $\operatorname{deg}(\lambda)<n$, we have $\mathfrak{S}_{\lambda}^{(n)}=s_{\lambda}$.

Proof. Given an $n$-core $\lambda$ where $\operatorname{deg}(\lambda)<n$ and a partition $\mu$ of length $r$ where $|\mu|=\operatorname{deg}(\lambda)$, we shall prove that there is a bijection between $A B C(\lambda, \mu)$ and $S S Y T(\lambda, \mu)$.

By Lemma 19, $A \in A B C(\lambda, \mu)$ is defined by a sequence of $n$-cores

$$
\emptyset \subset \lambda^{(1)} \subset \cdots \subset \lambda^{(r)}=\lambda
$$

where $\left(\lambda^{(i-1)}, \lambda^{(i)}\right)$ is a horizontal strong $\left(n-1-\mu_{i}\right)$-strip. Note in particular that $n-1-\mu_{i}=$ $n-1+\operatorname{deg}\left(\lambda^{(i-1)}\right)-\operatorname{deg}\left(\lambda^{(i)}\right)$. Since Theorem 14 implies that $w_{\lambda^{(i)}} w_{\lambda^{(i-1)}}^{-1}$ is cyclically decreasing of length $\ell\left(w_{\lambda^{(i)}}\right)-\ell\left(w_{\lambda^{(i-1)}}\right)=\mu_{i}$, there are $\mu_{i}$ distinct residues labelling the cells of the horizontal strip $\lambda^{(i)} / \lambda^{(i-1)}$ by Lemma 4. If $\lambda \in C^{n}$ with $\operatorname{deg}(\lambda)<n$, then no two cells that lie at the top of 
their column in $\lambda^{(i)} \subset \lambda$ can have the same $n$-residue. Therefore, $\lambda^{(i)} / \lambda^{(i-1)}$ is a horizontal $\mu_{i}$-strip, implying that (21) is an element of $S S Y T(\lambda, \mu)$.

On the other hand, given a semi-standard tableau $T \in S S Y T(\lambda, \mu)$ defined by (21), it suffices to show that $\left(\lambda^{(i-1)}, \lambda^{(i)}\right)$ is a horizontal strong $n-1-\mu_{i}$-strip for all $i$. By definition of semi-standard tableau, $\lambda^{(i)} / \lambda^{(i-1)}$ is a horizontal $\mu_{i}$-strip for $1 \leq i \leq r$. Since $\operatorname{deg}(\lambda)<n, \mu_{i}$ distinct residues

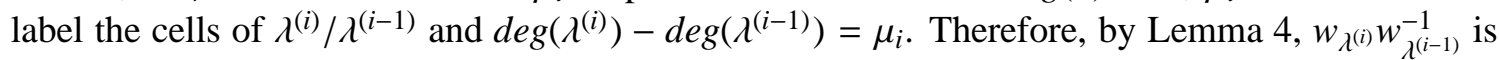
cyclically decreasing with length $\mu_{i}$. Theorem 14 then implies the result.

An $A B C$ countertableau $A$ comes equipped with a ribbon tiling specified by its defining strong strips. Let the column residue of every cell in column $c$ be $c-1(\bmod n)$. Recall that the bijection $\phi$ identifies a horizontal strong $(n-1-m)$-strip $\left(\lambda^{(i)}, \lambda^{(i+1)}\right)$ with a reduced word $j_{1} \cdots j_{m}$ for $w_{\lambda^{(i+1)}} w_{\lambda^{(i)}}^{-1}$ by a tiling of $R\left(n-1, \lambda^{(i)}\right) / \lambda^{(i+1)}$ with ribbons of height one that are determined by placing a tail in the rightmost cell of the bottom row with residue $t \in\{x-1, \ldots, x+1\} \backslash\left\{j_{1}, \ldots, j_{m}\right\}$ for $x=\lambda_{1}^{(i)}-1(\bmod n)$. Therefore, since $A$ is given by $\mu^{(0)} \subset \cdots \subset \mu^{(r)}$ where $\mu^{(r+1-i)}=$ $\left(\mu_{1}^{(r-i)}, \ldots, \mu_{r-i}^{(r-i)}, R\left(n-1, \lambda^{(i)}\right)\right)$, these ribbons tile row $r+1-i$ of $A$ where the residues of their tails are now column residues and ribbons containing letter $j>i$ in row $i$ are copies of the ribbons specified in row $j$.

A diagram derived from an $A B C A$ of weight $\mu \in \mathcal{P}^{n}$ called the extension ext $(A)$ is a useful tool to convert between affine factorizations and $A B C$ 's. It is formed by appending a ribbon of length $\lambda_{1}^{(x)}-\lambda_{1}^{(x-1)}+1$ to the end of row $x$, and then deleting any letter larger than $x$ in row $x$ and the tail of every ribbon containing $x$.

Example 23. For $n=6$ and an $A B C$ A of weight $(3,3,3,1)$, we have

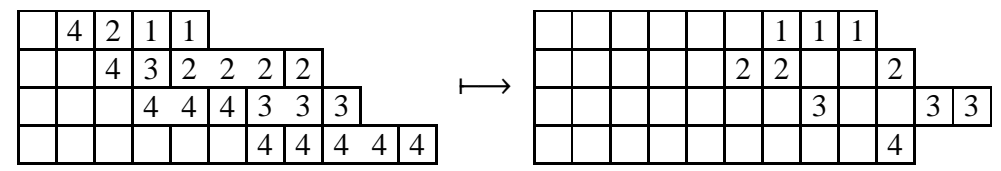

Lemma 24. For $A \in A B C(\lambda), j_{1} \cdots j_{\ell}$ is a reduced word for $v^{i}$ in the affine factorization $\Theta(A)=$ $v^{r} \cdots v^{1}$ if and only if the cells containing $i$ in ext $(A)$ have column residues $\left\{j_{1}, \ldots, j_{\ell}\right\}$.

Proof. Consider the $A B C$ given by $\mu^{(0)} \subset \cdots \subset \mu^{(r)}$ where

$$
\mu^{(r-i+1)}=\left(\lambda_{1}^{(r-1)}+n-1, \ldots, \lambda_{1}^{(i)}+n-1, R\left(n-1, \lambda^{(i-1)}\right)\right)
$$

and

$$
\mu^{(r-i)}=\left(\lambda_{1}^{(r-1)}+n-1, \ldots, \lambda_{1}^{(i)}+n-1, \lambda^{(i)}\right) .
$$

The parts of $\mu^{(r-i+1)}$ and $\mu^{(r-i)}$ differ only in the top $i$ rows where we find the cells of $A$ containing an $i$ forming the horizontal strip $D=R\left(n-1, \lambda^{(i-1)}\right) / \lambda^{(i)}$. In particular, the bottom row of $D$ in $A$ has an $i$ in columns $\lambda_{1}^{(i)}+1, \ldots, \lambda_{1}^{(i-1)}+n-1$. To determine which cells of $\operatorname{ext}(A)$ contain $i$, a ribbon of length $\lambda^{(i)}-\lambda^{(i-1)}+1$ is appended to the end of this row and we must appeal to the strong strips to delete the tails. 
Theorem 20 implies that $\Theta(A)=v^{r} \cdots v^{1}$ is an affine factorization for $w_{\lambda}$ where $v^{i}=w_{\lambda^{(i)}} w_{\lambda^{(i-1)}}^{-1}$. For each $i$, the proof of Theorem 19 uniquely identifies a reduced word $j_{1} \cdots j_{n-1-m}$ for $v^{i}$ with the strong chain

$$
\lambda^{(i)}=v^{(0)} \lessdot_{B} \cdots \lessdot_{B} v^{(m)}=R\left(n-1, \lambda^{(i-1)}\right),
$$

where $v^{(i-1)}$ is obtained from $v^{(i)}$ by deleting all removable copies of the ribbon whose tail has residue $a_{m-i+1}$ and lies in the bottom row, for the elements $x+1 \leq a_{m}<\cdots<a_{1} \leq x-1$ of $\{x-1, \ldots, x+1\} \backslash\left\{j_{1}, \ldots, j_{n-1-m}\right\}$ and $x=\lambda^{(i-1)}-1(\bmod n)$. Appending a ribbon of length $\lambda_{1}^{(i)}-\lambda_{1}^{(i-1)}+1$ to the end of the bottom row of $R\left(n-1, \lambda^{(i-1)}\right) / \lambda^{(i)}$ gives a skew shape where $\left\{j_{1}, \ldots, j_{n-1-m}\right\}$ is the set of column residues labeling the cells in the bottom row excluding ribbon tails.

\section{$6 t$-generalized affine Schubert polynomials}

Macdonald's basis of $P$-functions (see [Mac95]) are defined by

$$
P_{\lambda}(x ; t)=\frac{1}{v_{\lambda}(t)} \sum_{w \in S_{n}} w\left(x^{\lambda} \prod_{i<j} \frac{x_{i}-t x_{j}}{x_{i}-x_{j}}\right)
$$

where $v_{\lambda}(t)=\prod_{j \geq 0} \prod_{i=1}^{m_{j}} \frac{1-t^{i}}{1-t}$ for $m_{j}$ the multiplicity of $j$ in $\lambda$. For convenience, we work with the deformation $\tilde{P}_{\lambda}(x ; t)=t^{-n(\lambda)} P_{\lambda}\left(x ; t^{-1}\right)$ where $n(\lambda)=\sum_{i}(i-1) \lambda_{i}$. The set of $\tilde{P}$-functions forms a basis for $\Lambda$ that generalizes the monomial basis; when $t=1, \tilde{P}_{\mu}(x ; 1)=m_{\mu}$. One of the most important features of this basis is that the Kostka-Foulkes polynomials are inscribed in the Schur to $\tilde{P}$-function transition matrix:

$$
s_{\lambda}=\sum_{\mu} K_{\lambda \mu}(t) \tilde{P}_{\mu}(x ; t)
$$

Moreover, the $q=0$ case of the Macdonald polynomials $\left\{H_{\mu}(x ; 0, t)\right\}$ arise as the the dual basis to $\left\{\tilde{P}_{\mu}\right\}$ with respect to the Hall-inner product.

Lascoux and Schützenberger found an intrinsically positive formula for the Kostka-Foulkes polynomials by associating a statistic (non-negative integer) called cocharge to each semi-standard tableaux and proving that

$$
K_{\lambda \mu}(t)=\sum_{T \in S S Y T(\lambda, \mu)} t^{\operatorname{cocharge}(T)} .
$$

The cocharge of a standard tableau $T$ is the sum of the entries in the index vector $I(T)=\left[0, I_{2}, \ldots, I_{m}\right]$ which is defined by setting $I_{r}=I_{r-1}$ when the content of $r$ is larger than the content of $r-1$ and otherwise setting $I_{r}=I_{r-1}+1$. The notion is extended to give the cocharge of a semi-standard tableau with generic weight by successively computing the index of an appropriate subset of $i$ cells containing the letters $1,2, \ldots, i$.

Definition 25. From a specific $x$ in cell $c$ of a tableau $T$, the desired choice of $x+1$ is the southeasternmost one lying above $c$. If there are none above $c$, the choice is the south-easternmost $x+1$ in all of $T$. 
Consider now any semi-standard tableau $T$ with partition weight. Starting from the rightmost 1 in $T$, use Definition 25 to distinguish a standard sequence of $i$ cells containing $1,2, \ldots, i$. Compute the index and then delete all cells in this sequence. Repeat the process on the remaining cells. The total cocharge is defined to be the sum of all the index vectors.

Example 26. The cocharge of the following tableau is 25:

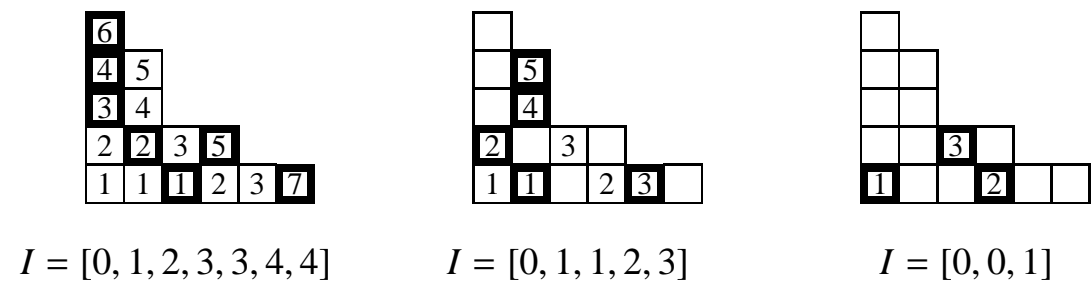

The $n$-cocharge of an $A B C$ depends on computing an index vector in a similar spirit. However, the role of $n$ brings forth an additional concept. Any ribbon in an $A B C$ that is filled with letter $i$ but does not lie in row $i$ is called an offset. The number of cells that are not tails in all the offsets,

$$
\text { off }(A)=\sum_{\text {R: offset in } A}(\operatorname{size}(R)-1),
$$

is one of the two components needed when computing $n$-cocharge. The index is the other, computed on the extension of $A$. Our construction of the index considers only the cells in $\operatorname{ext}(A)$. For $A$ of weight $1^{m}, \operatorname{ext}(A)$ is standard; there is exactly one cell in each row $i$ (coming from the single ribbon head with an $i$ in row $i$ of $A$ ). In this case, the $k$-cocharge is defined by computing an index vector $I=\left[0, I_{2}, \ldots, I_{m}\right]$ defined by

$$
I_{r+1}= \begin{cases}I_{r} & \text { when } r+1 \text { is east of } r \\ I_{r}+1 & \text { when } r+1 \text { is west of } r .\end{cases}
$$

Example 27. From the $A B C$ of weight $\left(1^{7}\right)$ :

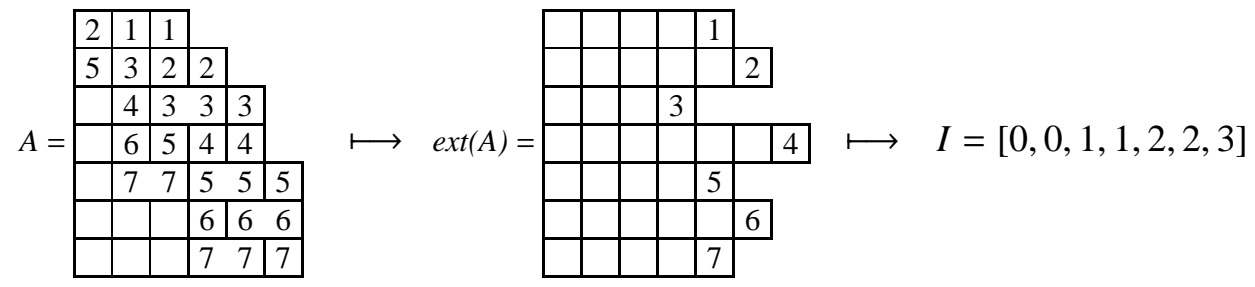

Equipped with a method to obtain the index when $\operatorname{ext}(A)$ has a single $i$ in row $i$, we describe a method for extracting standard fillings from an $A B C$ of arbitrary weight $\mu \in \mathcal{P}^{n}$.

Definition 28. Given an ABC A of weight $\mu \in \mathcal{P}^{n}$, consider its labelling by column residues. Iteratively earmark a standard sequence starting with the rightmost 1. From an $x$ (of column residue i) the appropriate choice of $x+1$ will be determined by choosing its column residue from the set $\mathcal{B}$ of all column residues labelling the $x+1$ 's. Reading counter-clockwise from $i$, this choice is the closest $j \in \mathcal{B}$ on a circle labelled clockwise with $0,1, \ldots, n-1$. 


\section{Example 29.}

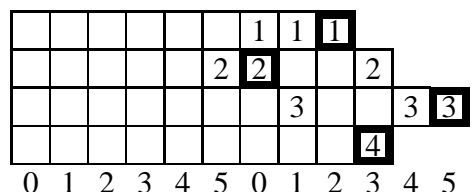

$I=[0,1,1,2]$

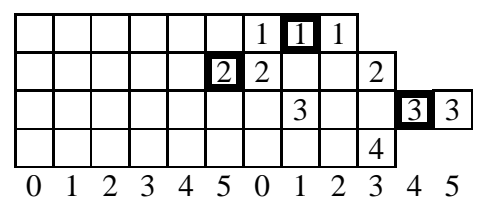

$I=[0,1,1]$

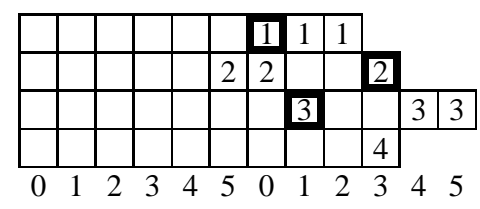

$I=[0,0,1]$

Definition 30. For an $A B C$ A of weight $\mu \in \mathcal{P}^{n}$, the $n$-cocharge of $A$ is defined by

$$
n-\operatorname{cocharge}(A)=\sum_{r} I_{r}(A)+\operatorname{off}(A) \text {. }
$$

We use the Schur expansion in $\tilde{P}$-functions $(23)$ as a guide to introduce a new family of symmetric functions involving the parameter $t$ that play a role in affine Schubert calculus and the theory of Macdonald polynomials.

Definition 31. For $\lambda \in \mathcal{P}^{n}$, set

$$
\Im_{c(\lambda)}^{(n)}(x ; t)=\sum_{\mu \in \mathcal{P}^{n}} K_{\lambda \mu}^{n}(t) \tilde{P}_{\mu}(x ; t),
$$

where the coefficients are taken to be the n-cocharge generating functions of $A B C$ 's (or weak Koskta-Foulkes polynomials),

$$
K_{\lambda \mu}^{n}(t)=\sum_{A \in A B C(\mathrm{c}(\lambda), \mu)} t^{n-\operatorname{cocharge}(A)} .
$$

For each $n>1$, consider the restricted linear span of Macdonald polynomials $H_{\lambda}(x ; 0, t)$ and the $\tilde{P}$-functions defined by

$$
\Lambda_{(n)}^{t}=\mathcal{L}\left\{H_{\lambda}(x ; 0, t): \lambda_{1}<n\right\} \quad \text { and } \quad \Lambda^{(n)^{t}}=\mathcal{L}\left\{\tilde{P}_{\lambda}(x ; t): \lambda_{1}<n\right\} .
$$

When $t=1$, these reduce to $\Lambda_{(n)}$ and $\Lambda^{(n)}$, respectively.

Proposition 32. For $n>1$, the set $\left\{\Im_{\lambda}^{(n)}(x ; t)\right\}_{\lambda \in C^{n}}$ forms a basis for $\Lambda^{(n)^{t}}$ that reduces to a set of representatives for the Schubert cohomology classes of Gr when $t=1$.

Proof. Since $\tilde{P}_{\mu}(x ; 1)=m_{\mu}$, we have that $\left\{\mathfrak{S}_{\lambda}^{(n)}(x ; 1)\right\}=\left\{\mathfrak{S}_{\lambda}^{(n)}\right\}$ which gives a set of Schubert representatives $\left\{\xi^{w_{\lambda}}\right\}$ in $H^{*}(\mathrm{Gr})$ by Theorem 20, The set $\left\{\tilde{P}_{\lambda}(x ; t): \lambda \in \mathcal{P}^{n}\right\}$ is linearly independent and therefore a basis for $\Lambda^{(n)^{t}}$. Therefore, $\mathfrak{S}_{\lambda}^{(n)}(x ; t) \in \Lambda^{(n)^{t}}$ implies that it suffices to show the transition matrix between $\tilde{P}$-functions and $\left\{\subseteq_{\lambda}^{(n)}(x ; t)\right\}_{\lambda \in C^{n}}$ is invertible. The matrix is square since there is a bijection between $n$-cores and elements of $\mathcal{P}^{n}$ and in fact invertible since Corollary 21 implies

$$
K_{\eta \mu}^{n}(t)=t^{n \text {-cocharge }(B)}+\sum_{\substack{A \in A B C(\mathrm{c}(\eta), \mu) \\ \eta \nless \mu}} t^{n \text {-cocharge }(A)}
$$

where $B$ is the unique $A B C$ of wieght $\eta$ and shape $\mathrm{c}(\eta)$. 
Let the set of functions $\left\{s_{\gamma}^{(n)}(x ; t)\right\}_{\gamma \in C^{n}}$ be the basis for $\Lambda_{(n)}^{t}$ defined by the duality relation, with respect to the Hall-inner product,

$$
\left\langle\Im_{\mu}^{(n)}(x ; t), s_{\gamma}^{(n)}(x ; t)\right\rangle=\delta_{\mu \gamma} .
$$

Since the Macdonald polynomial $H_{\lambda}(x ; q, t)$ reduces to the Hall-Littlewood polynomial $H_{\lambda}(x ; t)$ when $q=0$, we are now able to prove there is a natural tie between affine Schubert calculus and Macdonald polynomials.

Corollary 33. For each $n$-core $\lambda, s_{\lambda}^{(n)}(x ; 1)$ represents the Schubert class $\xi_{w_{\lambda}}$ in $H_{*}(G r)$ and for every $\mu \in \mathcal{P}^{n}$, the Macdonald polynomial at $q=0$ satisfies the non-negative expansion

$$
H_{\mu}(x ; 0, t)=\sum_{\lambda} K_{\lambda \mu}^{n}(t) s_{c(\lambda)}^{(n)}(x ; t) .
$$

Proof. The result follows by noting that (27) reduces to (17) when $t=1$ by Proposition 32 and that (17) defines the $k$-Schur functions representing the Schubert class $\xi_{w_{\lambda}}$.

Combinatorial results on $A B C$ 's can be used to prove that when $n>|\lambda|$, both $s_{\lambda}^{(n)}(x ; t)$ and $\mathfrak{S}_{\lambda}^{(n)}(x ; t)$ reduce to the Schur function $s_{\lambda}$.

Lemma 34. Given a partition $\lambda$ where $|\lambda|<n$, if $T$ is a semi-standard tableau of shape $\lambda$ then Definition 28 reduces to Definition 25

Proof. Consider $x$ of $n$-residue $i$ in $T$. Note that $|\lambda|<n$ implies that there is a unique cell $c$ of residue $i$ that contains $x$. Let $j$ be the first entry on the circle reading counter-clockwise from $i$ that is a residue of a cell containing $x+1$. If there is an $x+1$ above $c$, then the south-easternmost cell containing an $x+1$ that is above $c$ has residue $j$ since there are no $x+1$ 's of a residue counter-clockwise between $i$ and $j$. If there are none above $c$, then for the same reason, the south-easternmost cell containing an $x+1$ has residue $j$.

Proposition 35. For $\lambda \in C^{n}$ with $\operatorname{deg}(\lambda)<n, \mathfrak{S}_{\lambda}^{(n)}(x ; t)=s_{\lambda}^{(n)}(x ; t)=s_{\lambda}$.

Proof. Let $\lambda \in C^{n}$ with $\operatorname{deg}(\lambda)<n$. By Lemma 19, $A \in A B C(\lambda, \mu)$ is defined by a sequence of $n$-cores

$$
\emptyset \subset \lambda^{(1)} \subset \cdots \subset \lambda^{(r)}=\lambda
$$

where $\left(\lambda^{(i-1)}, \lambda^{(i)}\right)$ is a horizontal strong $\left(n-1-\mu_{i}\right)$-strip. This sequence is in one to one correspondence with a unique element $T \in S S Y T(\lambda, \mu)$ by Proposition 22. Lemmas 4 and 24 imply that the set of residues labelling cells of $\lambda^{(i)} / \lambda^{(i-1)}$ is the same as the set of column residues of cells containing the letter $i$ in $\operatorname{ext}(A)$. Since Definition 28 depends only on residues, it remains to show that the index vector on a standard sequence matches.

Note that the cocharge index of a standard sequence of $T$ can be defined by ordering the residues of $T$ with respect to

$$
x+1<x+2<\cdots<0<1<\cdots<x-1<x, \quad \text { where } x=\lambda_{1}^{(i)}-1 \quad(\bmod n),
$$


and setting $I_{i}=I_{i-1}$ when the residue of the cell containing $i$ is larger than the residue of $i-1$ and $I_{i}=I_{i-1}+1$ otherwise. Recall also that the index for an $A B C$ is computed by geographically comparing a cell containing $i-1$ in $\operatorname{ext}(A)$ to a cell containing $i$; if $i$ is east of $i-1$ then $I_{i}=I_{i-1}$ and $I_{i}=I_{i-1}+1$ otherwise. We claim that the colum residue of the cell containing $i$ is larger than that containing $i-1$ only when $i$ is east of $i-1$.

First we claim that if $\lambda_{1}^{(i-1)}<\lambda_{1}^{(i)}$, then there is no ribbon $S$ containing $i-1$ that has a non-tail cell in columns $\left[\lambda_{1}^{(i-1)}+1, \lambda_{1}^{(i)}\right]$ of $A$. By way of contradiction suppose such a ribbon $S$ containing $i-1$ with a non-tail cell $c$ of column residue $r$ exists in $A$. By Lemma 24, $\lambda^{(i-1)}$ has a cell of residue $r$. Since $c$ is in columns $\left[\lambda_{1}^{(i-1)}+1, \lambda_{1}^{(i)}\right]$, where $\lambda_{1}^{(i-1)}<\lambda_{1}^{(i)}$, then $\lambda^{(i)} / \lambda^{(i-1)}$ has a cell in the bottom row of residue $r$. Thus $T$ has two distinct cells, not on the same diagonal and of residue $r$. This contradicts the assumption that $\operatorname{deg}(\lambda)<n$.

Observe that the $(i-1)^{s t}$ row of $A$ has its rightmost cell in column $\lambda_{1}^{(i-2)}+n-1$. Since $\operatorname{ext}(A)$ is constructed by first appending $\lambda_{1}^{(i-1)}+\lambda_{1}^{(i-2)}+1$ cells to the $(i-1)^{s t}$ row of $A$, then the cells containing $i-1$ in $\operatorname{ext}(A)$ are within columns

$$
\left[\lambda_{1}^{(i-1)}+1,\left(\lambda_{1}^{(i-2)}+n-1\right)+\left(\lambda_{1}^{(i-1)}-\lambda_{1}^{(i-2)}+1\right)\right]=\left[\lambda_{1}^{(i-1)}+1, \lambda_{1}^{(i-1)}+n\right] .
$$

Similarly, the cells containing $i$ in $\operatorname{ext}(A)$ are within columns $\left[\lambda_{1}^{(i)}+1, \lambda_{1}^{(i)}+n\right]$. Since there are no ribbons containing $i-1$ that have a non-tail cell in columns $\left[\lambda_{1}^{(i-1)}+1, \lambda_{1}^{(i)}\right]$ of $A$, then the cells containing $i-1$ in $\operatorname{ext}(A)$ are actually within columns $\left[\lambda_{1}^{(i)}+1, \lambda_{1}^{(i-1)}+n\right]$. Since $\left[\lambda_{1}^{(i)}+1, \lambda_{1}^{(i-1)}+n\right] \subseteq$ $\left[\lambda_{1}^{(i)}+1, \lambda_{1}^{(i)}+n\right]$, then when $i$ is east of $i-1$ in $\operatorname{ext}(A)$, its column residue is larger with respect the same ordering (29).

It remains to prove that of $f(A)=0$. By contradiction, if off $f(A)>0$ then $A$ has a ribbon $O$ of length greater than 1 and filled with the letter $i$ that is not in the $i^{\text {th }}$ row, for some $1 \leq i \leq \ell(\mu)$. On the one hand, note that $O$ has cells which are at the top of their columns in $R\left(n-1, \lambda^{(i-1)}\right)$, and not in the bottom row. There is a horizontal ribbon in the bottom row of $R\left(n-1, \lambda^{(i-1)}\right)$ whose cells are at the top of their columns and of the same residue as those of $O$. By Lemmas 24, the set of residues labeling cells of $\lambda^{(i)} / \lambda^{(i-1)}$ is the same as the set of column residues of cells containing letter $i$ in $\operatorname{ext}(A)$. Thus, in $R\left(n-1, \lambda^{(i-1)}\right)$, the residues of non-tail cells of $O$ are the same as the residues labeling a horizontal strip $S$ in $\lambda^{(i)} / \lambda^{(i-1)}$.

On the other hand, if $\lambda \in C^{n}$ with $\operatorname{deg}(\lambda)<n$, then no two cells that lie at the top of their columns in $\lambda^{(i)} \subset \lambda$ can have the same $n$-residue. Thus the residues labeling cells of $\lambda^{(i)} / \lambda^{(i-1)}$ are unique. Since $O$ is not in the bottom row of $R\left(n-1, \lambda^{(i-1)}\right)$, and it is of length greater than 1 , then it must be the case that there is a non-tail cell of it which is above a cell of $S$. This contradicts the fact that $R\left(n-1, \lambda^{(i-1)}\right) / \lambda^{(i-1)}$ is a horizontal strip; constructed by adding a cell to the top of every column of $\lambda^{(i-1)}$ and $n-1$ cells to its bottom row.

\section{Quantum cohomology of flags}

Here we show that the strong formulation of the Pieri rule for $H_{*}(\mathrm{Gr})$ given in Corollary 15 can be applied to the problem of computing intersections in the (small) quantum cohomology of a flag 
variety. The examination leads to a distinguished family of strong chains defined by a notion of translation that generalizes $R(n-1, \lambda)$.

In this section, we switch to representing the indices of Schubert basis elements by partitions $\lambda \in \mathcal{P}^{n}$. Recall that $R(n-1, \lambda)$ was defined to be $\left(\lambda_{1}+n-1, \lambda\right)$ when $\lambda$ is an $n$-core in $\S 4.2$. Here, we abuse notation and instead define $R(n-1, \eta)=\left(\mathfrak{c}(\eta)_{1}+n-1, \mathfrak{c}(\eta)\right)$ for $\eta \in \mathcal{P}^{n}$.

\subsection{An affine Monk formula}

The quantum cohomology ring $Q H^{*}(X)$ is defined for any Kähler algebraic manifold $X$, but we consider only the complete flag manifold $X=F l_{n}$ of chains of vector spaces in $\mathbb{C}^{n}$. The quantum cohomolgy ring is simply $Q H^{*}\left(F l_{n}\right)=H^{*}\left(F l_{n}\right) \otimes \mathbb{Z}\left[q_{1}, \ldots, q_{n-1}\right]$ for parameters $q_{1}, \ldots, q_{n-1}$ as a linear space but the multiplicative structure is much richer than the specialization of $q_{1}=\cdots=$ $q_{n-1}=0$. Cells in the Schubert decomposition of $Q H^{*}\left(F l_{n}\right)$ are indexed by permutations $w \in S_{n}$, and the quantum product is defined by

$$
\sigma_{u} * \sigma_{v}=\sum_{w} \sum_{d} q_{1}^{d_{1}} \ldots q_{n-1}^{d_{n-1}}\langle u, v, w\rangle_{d} \sigma_{w_{0} w},
$$

where the structure constants are 3-point Gromov-Witten invariants of genus 0 which count equivalence classes of certain rational curves in $F l_{n}$. The understanding and computation of GromovWitten invariants is a widely studied problem.

Although the construction is not manifestly positive, all 3-point, genus zero Gromov-Witten invariants $\langle u, w, v\rangle_{d}$ in (30) can be computationally obtained from the subset

$$
\left\{\left\langle s_{r}, w, v\right\rangle_{d}: 1 \leq r<n \text { and } w, v \in S_{n}\right\}
$$

since the quantum cohomology is generated by the codimension one Schubert classes. By defining a family of quantum Schubert polynomials, Fomin, Gelfand, and Postnikov were able to prove that there is a simple combinatorial characterization for this set that generalizes the classical Monk formula [Mon59].

Theorem 36. [FGP97] (Quantum Monk formula) For $w \in S_{n}$ and $1 \leq r<n$, the quantum product of the Schubert classes $\sigma_{s_{r}}$ and $\sigma_{w}$ is given by

$$
\sigma_{s_{r}} * \sigma_{w}=\sum \sigma_{w \tau_{a, b}}+\sum q_{c} q_{c+1} \cdots q_{d-1} \sigma_{w \tau_{c, d}}
$$

where the first sum is over all transpositions $\tau_{a, b}$ such that $a \leq r<b$ and $\ell\left(w \tau_{a, b}\right)=\ell(w)+1$, and the second sum is over all transpositions $\tau_{c, d}$ such that $c \leq r<d$ and $\ell\left(w \tau_{c, d}\right)=\ell(w)-\ell\left(\tau_{c, d}\right)=$ $\ell(w)-2(d-c)+1$.

We have found that the idea of horizontal strong strips extends to include combinatorics of the flag Gromov-Witten invariants and the quantum Monk rule. Peterson asserted that $Q H^{*}(G / P)$ of a flag variety is, up to localization, a quotient of the homology $H_{*}\left(\mathrm{Gr}_{G}\right)$ of the affine Grassmannian 
$\mathrm{Gr}_{G}$ of $G$ (proven in [LS12]). As a consequence, the Gromov-Witten invariants arise as homology Schubert structure constants of $H_{*}\left(\mathrm{Gr}_{G}\right)$. The identification of $\langle u, v, w\rangle_{d}$ with the coefficients in

$$
\xi_{\mu} \xi_{\lambda}=\sum_{v} c_{\mu, \lambda}^{v} \xi_{v}
$$

was made explicit in $[\mathrm{LM}]$. The identification hinges on a correspondence between permutations in $S_{n}$ and certain partitions defined by

$$
\operatorname{sh}: w \mapsto \lambda \quad \text { for } \quad \lambda_{i}^{\prime}=\left(\begin{array}{c}
n-i \\
2
\end{array}\right)+i n v_{i}\left(w_{0} w\right),
$$

where $\lambda^{\prime}$ is the partition obtained by reflecting the shape of $\lambda$ about the line $y=x$, the inversion $\operatorname{inv}_{i}(u)$ is the number of $u_{j}<u_{i}$ for $i<j$, and $w_{0}=[n, n-1, \ldots, 1]$ is the permutation of maximal length in $S_{n}$.

Theorem 37. $[L M]$ For $u, v, w \in S_{n}$ and $d \in \mathbb{N}^{n-1}$,

$$
\langle u, w, v\rangle_{d}=c_{s h(u), s h(w)}^{\eta},
$$

where $\eta$ is obtained by adding $\left(\begin{array}{c}n+1-i \\ 2\end{array}\right)-(n-i+1) d_{i}+(n-i) d_{i-1}$ cells to column i of $\operatorname{sh}(v)$, for $1 \leq i<n$.

The image of $S_{n}$ under the map sh is the set of $n$ ! partitions $\mathcal{P}_{\square}^{n}=\{\lambda: \square / \lambda=$ vertical strip $\}$, where the partition $\square=\left(n-1, n-2^{2}, \ldots, 1^{n-1}\right)$. This foreshadows that $(n-1)$-rectangles, the shapes $R_{r}=\left(r^{n-r}\right)$ with $n-r$ rows of length $r$, play a role in the combinatorics of quantum cohomology of flag varieties as they have in various contexts of affine Schubert calculus (e.g. [LLM03, LM03, Mag07, LS12, BBTZ12]). At the root, for any partition $\lambda \in \mathcal{P}^{n}$ and $1 \leq r<n$,

$$
\xi_{\lambda \cup R_{r}}=\xi_{R_{r}} \xi_{\lambda} .
$$

Since a defining subset of Gromov-Witten invariants is given by (31), looking closely at $\operatorname{sh}\left(s_{r}\right)$ reveals the role of these shapes in the combinatorics of quantum cohomology. To be precise, it was shown in [LM] that for any $v, w \in S_{n}$ and $1 \leq r<n$,

$$
\left\langle s_{r}, v, w\right\rangle_{d}=c_{R_{r}^{\prime}, \operatorname{sh}(w)}^{\eta \cup \operatorname{sh}(v) \cup R_{r}},
$$

where the $i$ th column of $\eta$ is $(n-i) d_{i-1}-(n+1-i) d_{i}$ and $R_{r}^{\prime}$ is the shape obtained by deleting the corner box from $R_{r}$. In particular, Monk's classical formula is determined by $c_{R_{r}^{\prime} \text {, sh }(v)}^{\operatorname{sh}(w)}$. Therefore, for any $u, v, w \in S_{n}$, all Gromov-Witten invariants $\langle u, v, w\rangle_{d}$ can be computed from the set

$$
\left\{c_{R_{r}^{\prime}, \lambda}^{v}: 1 \leq r<n, \lambda \in \mathcal{P}_{\square}^{n} \text {, and } v_{1}<n\right\} .
$$

The $\eta \cup \operatorname{sh}(v) \cup R_{r}$ in (37) suggests that the formula for these invariants is related to elements covered by the generic translation of $\lambda$ defined by

$$
R(r, \lambda)=\mathfrak{c}\left(\lambda \cup R_{r}\right) .
$$


Conjecture 38. (Affine Monk formula) For $1 \leq r<n$ and partition $\lambda$ with $\lambda_{1}<n$,

$$
\xi_{R_{r}^{\prime}} \xi_{\lambda}=\sum_{c(v)<_{B} R(r, \lambda)} \xi_{v}
$$

where $\mathfrak{c}(v)_{i}<R(r, \lambda)_{i}$ for some $i$ such that $\left(\lambda \cup R_{r}\right)_{i}=r$.

Note that the expansion (39) can be derived from results in [BSS13] that determine the expansion of a non-commutative $k$-Schur function indexed by $R_{r}^{\prime}$ in terms of words in the affine nilCoxeter $\operatorname{algebra} \mathbb{A}$.

Example 39. For $n=5, \lambda=(3,2,1,1)$, and $R_{3}^{\prime}=(3,2)$, term $\xi_{v}$ occurs in the expansion of $\xi_{R_{3}^{\prime}} \xi_{\lambda}$ when $v$ is a partition where $\mathfrak{c}(v) \lessdot_{B} R(3, \lambda)$ and $\mathfrak{c}(v)_{i}<R(3, \lambda)_{i}$ for some $i \in\{1,2,3\}$.

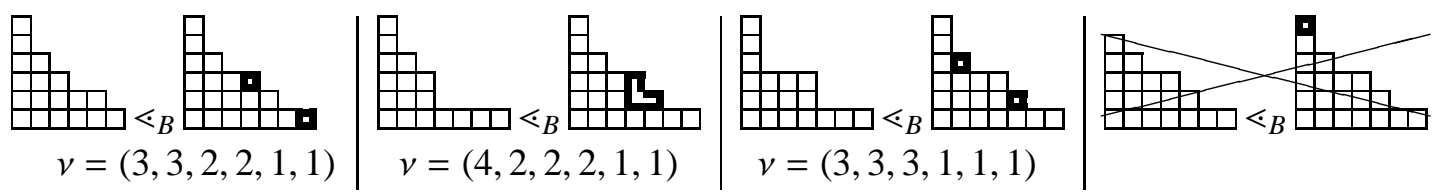

Relation (36) then gives the terms in the expansion of $\xi_{R_{3}^{\prime}} \xi_{\lambda \cup R_{r}}$ for any $R_{r}$. In particular,

$$
\xi_{R_{3}^{\prime}} \xi_{\lambda \cup R_{2}}=\xi_{(3,3,2,2,1,1) \cup R_{2}}+\xi_{(4,2,2,2,1,1) \cup R_{2}}+\xi_{(3,3,3,1,1,1) \cup R_{2}} .
$$

Since $\operatorname{sh}([4,2,5,3,1])=\lambda \cup R_{2} \in \mathcal{P}_{\square}^{n}$, this matches the quantum Monk expansion by Equation (37):

$$
\sigma_{s_{3}} * \sigma_{[4,2,5,3,1]}=\sigma_{[4,3,5,2,1]}+q_{3} \sigma_{[4,2,3,5,1]}+q_{3} q_{4} \sigma_{[4,2,1,3,5]} .
$$

\subsection{Ribbon strong strips}

When $r=n-1$, Conjecture 38 reduces to a special case of the expansion given in Corollary 15 , The terms are defined by horizontal strong 1-strips, which are in fact the elements $v$ covered by $R(n-1, \lambda)$ where $\mathfrak{c}(v)_{1}<R(n-1, \lambda)_{1}$. Horizontal strong strips of generic length $1 \leq b<n-1$ describe the expansion of $\xi_{(n-1-b)} \xi_{\lambda}$. We thus turn to the more general expansion, for $1 \leq b<r<n$ and partition $\lambda \in \mathcal{P}^{n}$,

$$
\xi_{\left(r^{n-1-r}, r-b\right)} \xi_{\lambda}=\sum_{v \in \mathcal{B}_{r, b, \lambda}} \xi_{v}
$$

as a guide to characterize a larger family of strong strips associated to the general translation $R(r, \lambda)$.

While horizontal strong strips are certain shapes differing from $R(n-1, \lambda)$ by a horizontal strip, the more general picture involves shapes that differ from $R(r, \lambda)$ by a horizontal ribbon strip, a sequences of shapes $v=v^{(0)} \subset v^{(1)} \subset \cdots \subset v^{(m)}$ such that $v^{(i)} / v^{(i-1)}$ is comprised of ribbons whose heads lie above a cell in $v$ (or in the bottom row), for all $1 \leq i \leq m$. Rather than requiring that bottom row lengths are increasing as we did for horizontal strong strip, we now require that a ribbon tail lies in a specified set of columns. For $1 \leq r<n$ and a partition $\lambda$ with $\lambda_{1}<n$, let $\eta=\lambda \cup R_{r}$. Let $m$ be the highest row of $\eta$ that has length $r$ and denote the set of $r$ columns containing the last $r$ cells in row $m$ of $\mathfrak{c}(\eta)$ by $\operatorname{col}_{r}(\lambda)$. 
Definition 40. Given $n$-cores $\lambda$ and $v$ and $1 \leq r<n$, the pair $(\lambda, v)$ is a ribbon strong strip with respect to $r$ if there is a horizontal ribbon strip

$$
v=v^{(0)} \lessdot_{B} v^{(1)} \lessdot_{B} \cdots \lessdot_{B} v^{(m)}=R(r, \lambda)
$$

with a ribbon tail of $v^{(i)} / v^{(i-1)}$ lying in $\operatorname{col}_{r}(\lambda)$ for all $i>0$. Its length is defined to be $m$.

The expansion [41) can be derived from results in [BSS] that determine the expansion of a non-commutative $k$-Schur function indexed by $\left(r^{n-1-r}, b\right)$ in terms of words in the affine nilCoxeter algebra $\mathbb{A}$. We instead conjecture that the expansion is simply the sum over $v$ such that $(\mathfrak{c}(\lambda), \mathfrak{c}(v))$ is a ribbon strong strip of length $b$ with respect to $r$.

Example 41. For $n=5$ and $\lambda=(4,2)$, the ribbon strong strips of length 2 with respect to $r=3$ are

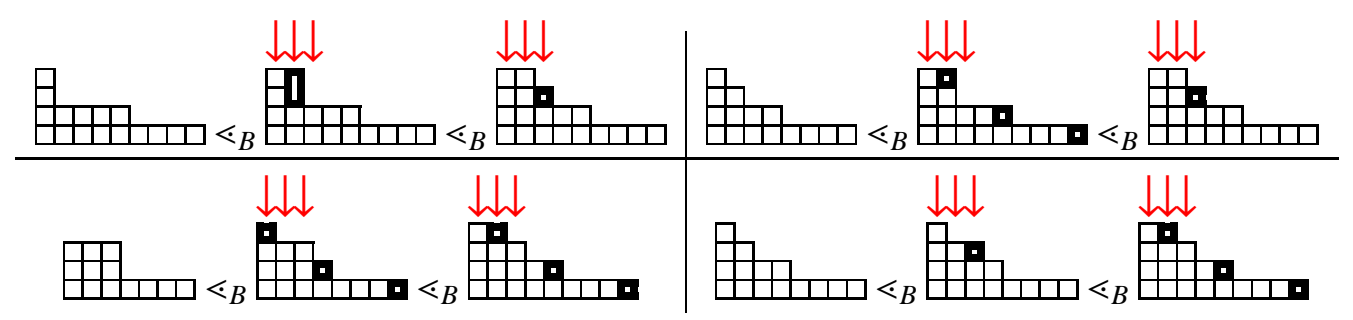

Conjecturally, this gives the expansion

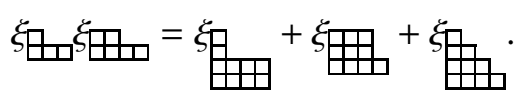

Conjecture 42. Given $n$-cores $\lambda$ and $v$ and $1 \leq r<n$, the pair $(\lambda, v)$ is a ribbon strong strip with respect to $r$ if and only if there exists a strong strip

$$
v=v^{(0)} \lessdot_{B} v^{(1)} \lessdot_{B} \cdots \lessdot_{B} v^{(m)}=R(r, \lambda)
$$

with a ribbon tail of $v^{(i)} / v^{(i-1)}$ lying in $\operatorname{col}_{r}(\lambda)$ for all $i>0$.

Proposition 43. Given n-cores $\lambda$ and $v,(\lambda, v)$ is a horizontal strong strip if and only if $(\lambda, v)$ is a ribbon strong strip with respect to $n-1$.

Proof. Let $p$ be the number of rows of length $n-1$ in $p(\lambda)$ and thus the bottom $p+1$ rows of $\mathfrak{p}(\lambda) \cup R_{n-1}$ have length $n-1$. Note by definition of $\mathfrak{c}$ that the last $n-1$ cells in rows $1, \ldots, p+1$ of $\mathfrak{c}\left(\mathfrak{p}(\lambda) \cup R_{n-1}\right)$ lie at the top of a column and have residues $\lambda_{1}, \lambda_{1}+1, \ldots, \lambda_{1}+(n-2)(\bmod n)$. Further, $\operatorname{col}_{n-1}(\lambda)$ is defined by taking the last $n-1$ columns in row $p+1$.

Assume $(\lambda, v)$ is a horizontal strong strip. Lemma 8 implies that $v^{(j)} / v^{(j-1)}$ consists of all copies of $S^{j}$ that can be removed from $v^{(j)}$ where $S^{j}$ is a removable ribbon in the bottom row of $v^{(j)}$. For $j=m$, the discussion in the previous paragraph implies that a copy of $S^{m}$ lies in row $p+1$. By iteration, a copy of $S^{j}$ (and in particular, its tail) lies in the last $n-1$ columns of row $p+1$ for all $j=1, \ldots, m$. 
On the other hand, consider a chain of $n$-cores $v=v^{(0)} \lessdot_{B} v^{(1)} \lessdot_{B} \cdots \lessdot_{B} v^{(m)}=R(n-1, \lambda)$ where the tail of a ribbon $S^{j}$ in $v^{(j)} / v^{(j-1)}$ lies in one of the last $n-1$ columns in row $p+1$ of $R(n-1, \lambda)$. Since the number of cell in $S^{j}$ is smaller than $n$ and there are $n-1$ cells at the top of a column in row $p, S^{m}$ must have height one. Therefore, it can be removed from every row $1, \ldots, p+1$ of $R(n-1, \lambda)$. By iteration, there is a copy of $S^{j}$ in the bottom row of $\left(n-1+\lambda_{1}, \lambda\right)$ for $j=1, \ldots, m$. Since the tail of $S^{1}$ is on of the last $n-1$ cells in row $p+1$ of residue $\lambda_{1}, \ldots, \lambda_{1}+(n-2) \bmod n$, $\lambda \subset v$.

Proposition 44. For $1 \leq r<n$ and for $n$-cores $\lambda$ and $v$, a ribbon strong strip $(\lambda, v)$ with respect to $r$ has length one if and only if $v \ll_{B} R(r, \lambda)$ and $v_{i}<R(r, \lambda)_{i}$ for some $i$ such that $\left(\lambda \cup R_{r}\right)_{i}=r$.

Proof. For $\eta \in \mathcal{P}^{n}$, let $\lambda=\mathfrak{c}(\eta)$ and let $m$ be the highest row of length $r$ in $R_{r} \cup \eta$. Note that the highest cell of $R(r, \lambda)$ in the leftmost column of $\operatorname{col}_{r}(\lambda)$ lies in a row no higher than row $m+(n-1-r)$ and that rows $m, m-1, \ldots, m-(n-1-r)$ of $R_{r} \cup \eta$ have length $r$.

Given $(\lambda, v)$ is a ribbon strong strip with respect to $r$ of length one, $v \ll_{B} R(r, \lambda)$ and the tail of a ribbon $S \subset R(r, \lambda) / v$ lies in a column of $\operatorname{col}_{r}(\lambda)$. Suppose the head of $S$ lies in row $a$. If $a \leq m$, then $v \leftarrow_{B} R(r, \lambda)$ and $v_{i}<R(r, \lambda)_{i}$ for some $i$ such that $\left(\lambda \cup R_{r}\right)_{i}=r$. When $a>m$, all cells of $S$ lie in colums of $\operatorname{col}_{r}(\lambda)$ and therefore in rows between $m+1$ and $m+n-1-r$. Proposition 9 [LM04] ensures that a removable copy of $S$ also lies in rows $m-(n-1-r), \ldots, m-1, m$, and the forward direction thus follows from Lemma 3 ,

On the other hand, consider $n$-cores $\lambda$ and $v$ such that $v_{i} \ll_{B} R(r, \lambda)_{i}$ for some $i$ where $\left(\lambda \cup R_{r}\right)_{i}=r$. In particular, there is a ribbon $S \subset R(r, \lambda) / v$ containing at least one cell in row $i$. If the tail $t$ of $S$ is not in a column of $\operatorname{col}_{r}(\lambda)$ then $t$ lies in row $i<m$. By Proposition 9 [LM04], there is an extremal cell $\tilde{t}$ of the same residue as $t$ that lies in a column of $\operatorname{col}_{r}(\lambda)$. Consider the subset $\tilde{S}$ of extremal cells in $R(r, \lambda)$ that is formed by taking all extremal cells between $\tilde{t}$ (as the highest) and a cell $\tilde{h}$ of the same residue as the head of $S$. Since $S$ can be removed from $R(r, \lambda)$, its head lies at the end of its row. Property 1 then implies that $\tilde{h}$ is at the end of its row and thus $\tilde{S}$ is a removable ribbon. By Lemma $3, \tilde{S} \subset R(r, \lambda) / v$ proving the claim.

\section{References}

[BB96] A. Björner and F. Brenti. Affine permutations of type A. The Foata Festsrift Electron, 18(35), 1996.

[BBPZ12] C. Berg, N. Bergeron, S. Pon, and M. Zabrocki. Expansions of $k$-Schur Functions in the Affine nil-Coxeter Algebra. The Electronic Journal of Combinatorics, 19(2), 2012.

[BBTZ12] C. Berg, N. Bergeron, H. Thomas, and M. Zabrocki. Expansion of $k$-Schur functions for maximal $k$-rectangles within the affine nil-Coxeter algebra. preprint, 2012.

[BKPT] A. Buch, A. Kresch, K. Purboo, and H. Tamvakis. The puzzle conjecture for the cohomology of two-step flag manifolds. arXiv:1401.1725. 
[BKT03] A. Buch, A. Kresch, and H. Tamvakis. Gromov-Witten invariants on Grassmannians. J. Amer. Math. Soc., 16(4):901-915, 2003.

[Bot58] R. Bott. The space of loops on a Lie group. Michigan Math. J., 5:35-61, 1958.

[BS98] N. Bergeron and F. Sottile. Schubert polynomials, the Bruhat order, and the geometry of flag manifolds. Duke Math. J., 95:373-423, 1998.

[BSS] Chris Berg, Franco Saliola, and Luis Serrano. Combinatorial expansions for families of non-commutative $k$-Schur functions. math.arXiv:1208.4857.

[BSS13] Chris Berg, Franco Saliola, and Luis Serrano. The down operator and expansions of near rectangular $k$-Schur functions. J. Combin. Theory Ser. A, 3:623-636, 2013.

[Buc] A. Buch. Mutations of puzzles and equivariant cohomology of two-step flag varieties. arXiv:1401.3065.

[Cos09] I. Coskun. A Littlewood-Richardson rule for two-step flag varieties. Invent. Math., 176(2):325-395, 2009.

[DM13] Avinash Dalal and Jennifer Morse. ABC's of the affine Grassmannian. DMTCS Proceedings, 2013.

[FGP97] S. Fomin, S. Gelfand, and A. Postnikov. Quantum Schubert polynomials. J. Amer. Math. Soc., 10(3):565-596, 1997.

[FK13] S. Fishel and M. Konvalinka. Results and conjectures on the number of standard strong marked tableaux. DMTCS Proceedings, 2013.

[GH96] A. Garsia and M. Haiman. A remarkable $q$, $t$-Catalan sequence and $q$-Lagrange inversion. J. Algebraic Combin., 5(3):191-244, 1996.

[GP92] A. M. Garsia and C. Procesi. On certain graded $S_{n}$-modules and the $q$-Kostka polynomials. Adv. Math., 87:82-138, 1992.

[GR75] H. Garland and S. Raghunathan. A Bruhat decomposition for the loop space of a compact group: a new approach to results of Bott. Proc. Nat. Acad. Sci., 72:47164717, 1975.

[Gre55] J. A. Green. The characters of the finite general linear groups. Trans. Amer. Math. Soc., 80:442-407, 1955.

[Hai01] M. Haiman. Hilbert schemes, polygraphs, and the Macdonald positivity conjecture. J.A.M.S., 14:941-1006, 2001.

[Hai08] M. Haiman. Combinatorics of skew-linked partitions. Notes from AMS Western Section Meeting, 2008. 
[HMZ12] J. Haglund, J. Morse, and M. Zabrocki. A compositional shuffle conjecture specifying touch points of the Dyck path. Canadian Journal of Math., 64(4):822-844, 2012.

[Jin91] N. Jing. Vertex operators and Hall-Littlewood symmetric functions. Adv. in Math., 87(2):226-248, 1991.

[Kac90] V. Kac. Infinite-dimensional Lie Algebras. Univ. Press, Cambridge, third edition, 1990.

[KK86] B. Kostant and S. Kumar. The nil-Hecke ring and cohomology of $G / P$ for a Kac-Moody group G. Adv. in Math., 62(3):187-237, 1986.

[KS10] C. Korff and C. Stroppel. The $\widehat{\mathrm{sl}}(n)_{k}-\mathrm{WZW}$ fusion ring: a combinatorial construction and a realization as quotient of quantum cohomology. Adv. in Math., 225(1):20-268, 2010 .

[KT03] A. Knutson and T. Tao. Puzzles and (equivariant) cohomology of Grassmannians. Duke Math. J., 119(2):221-260, 2003.

[Lam06] T. Lam. Affine Stanley symmetric functions. Amer. J. of Math., 128(6):1553-1586, 2006.

[Lam08] T. Lam. Schubert polynomials for the affine Grassmannian. J. Amer. Math. Soc., 21(1):259-281, 2008.

[Las99] A. Lascoux. Ordering the affine symmetric group. Algebraic combinatorics and applications (Gößweinstein, pages 219-231, 1999.

[LLM03] L. Lapointe, A. Lascoux, and J. Morse. Tableau atoms and a new Macdonald positivity conjecture. Duke Math. J., 116:103-146, 2003.

[LLMS10] T. Lam, L. Lapointe, J. Morse, and M. Shimozono. Affine insertion and Pieri rules for the affine Grassmannian. Memoirs of the AMS, 208(977), 2010.

[LLMS12] T. Lam, L. Lapointe, J. Morse, and M. Shimozono. The poset of k-shapes and branching of $k$-Schur functions. To appear in Memoirs of the AMS, 2012.

[LM] L. Lapointe and J. Morse. Flag Gromov-Witten invariants and $k$-Littlewood Richardson coefficients. preprint.

[LM03] L. Lapointe and J. Morse. Schur function analogs and a filtration for the symmetric function space. J. Combin. Theory Ser. A, 101(2):191-224, 2003.

[LM04] L. Lapointe and J. Morse. Order ideals in weak subposets of Young's lattice and associated unimodality conjectures. Ann. Combin., 8(2):197-219, 2004.

[LM05] L. Lapointe and J. Morse. Tableaux on $k+1$-cores, reduced words for affine permutations, and $k$-Schur function expansions. J. Combin. Theory Ser., 112(1):44-81, 2005. 
[LM07] L. Lapointe and J. Morse. A $k$-tableaux characterization for $k$-Schur functions. Adv. in Math., 213(1):183-204, 2007.

[LM08] L. Lapointe and J. Morse. Quantum cohomology and the $k$-Schur basis. Trans. Amer. Math. Soc., 360(4):2021-2040, 2008.

[LR34] D. E. Littlewood and A. R. Richardson. Group characters and algebra. Philos., 233:99_ $141,1934$.

[LS78] A. Lascoux and M.-P. Schützenberger. Sur une conjecture de H.O. Foulkes. C.R. Acad. Sc. Paris, 294:323-324, 1978.

[LS82] A. Lascoux and M.-P. Schützenberger. Polynômes de Schubert. C. R., 294(13):447$450,1982$.

[LS12] T. Lam and M. Shimozono. From quantum Schubert polynomials to $k$-Schur functions via the Toda lattice. To appear in Math Research Letters, 2012.

[Lus81] G. Lusztig. Singularities, character formulas, and a $q$-analog of weight multiplicities. Analysis and topology on singular spaces, II, III (Luminy), pages 101-102, 1981.

[Mac88] I. G. Macdonald. A new class of symmetric functions. Séminaire Lotharingien de Combinatoire, B20a 41pp, 1988.

[Mac95] I. G. Macdonald. Symmetric functions and Hall polynomials. Clarendon Press, Oxford, 2nd edition, 1995.

[Mag07] Peter Magyar. Notes on Schubert classes of a loop group. Technical report, preprint, 2007.

[Mon59] D. Monk. The geometry of flag manifolds. Proceedings of the London Mathematical Society, 2:253-186, 1959.

[MPP] K. Meszaros, G. Panova, and A. Postnikov. Schur times Schubert via the FominKirillov algebra. arXiv:1210.1295 [math.CO].

[MPS77] Marcel-Paul Marcel-Paul Schtzenberger. La correspondance de Robinson. Combinatoire et reprsentation du groupe symtrique (Actes Table Ronde CNRS, Univ. LouisPasteur Strasbourg, Strasbourg, 1976), pages 59-113, 1977.

[MS] Jennifer Morse and Anne Schilling. Crystal operators and flag Gromov-Witten invariants. preprint.

[MS12] Jennifer Morse and Anne Schilling. WZW fusion rule for break point triples. DMTCS Proceedings, 2012. 
[NY97] A. Nakayashiki and Y. Yamada. Kostka polynomials and energy functions in solvable lattice models. Selecta Math, 3:547-599, 1997.

[Pet97] D. Peterson. Lecture notes. M.I.T., 1997.

[PL] Maria-Elena Pinto and Luc Lapointe. Charge on tableaux and the poset of $k$-shapes. arXiv:1305.2438 [math.CO].

[Pos99] A. Postnikov. On a quantum version of Pieri's formula. Advances in Geometry, Progress in Mathematics, 172:371-383, 1999.

[Sta84] R. Stanley. On the number of reduced decompositions of elements in Coxeter groups. European J. Combin., 5:359-372, 1984.

[Tud00] G. Tudose. A special case of $S L(n)$-fusion coefficients. Technical report, preprint, 2000 .

[TUY89] A. Tsuchiya, K: Ueno, and Y. Yamada. Conformal field theory on universal family of stable curves with gauge symmetries. Adv. Stud. in Pure Math., 19:459-566, 1989.

[Ver88] E. Verlinde. Fusion rules and modular transformations in 2D conformal field theory. Nuclear Phys. B, 300(3):360-376, 1988.

[Wa190] M. Walton. Fusion rules in Wess-Zumino-Witten models. Nuclear Phys. B, 340:777$790,1990$.

[Zab98] Mike Zabrocki. On the action of the Hall-Littlewood vertex operator. University of California, San Diego, 1998. Ph.D. Thesis. 